|RAC| Academic Scientific Jourmals
Samarra Journal of Pure and Applied Science

www.sjpas.com

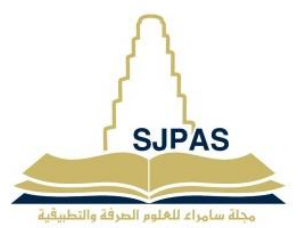

ISSN:2663-7405

\title{
تحضير وتثخيص بعض مركبات اميدات السلفا ودراسة فعاليتها المضادة للبكتريا
}

ميمونه حميد خضير 1* ، عبدالمجيد صالح حمد22 ، اسيل مقداد حاتم3 3

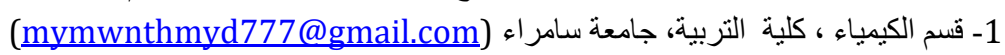

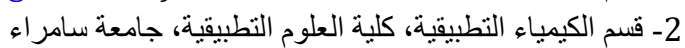

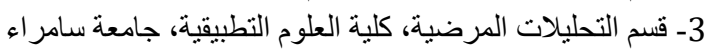
البحث مستل من رسالة ماجستير الباحث الأول البرل

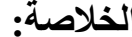

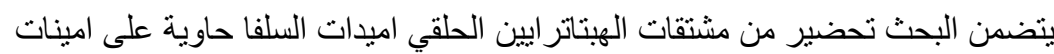

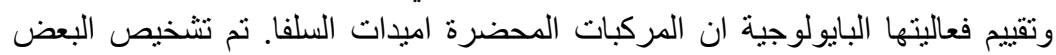

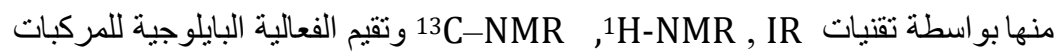
فقد نم القياس على اساس قياس قطر التثبيط الذي تسبيهُ العزلات البكات البكتيريه على الوسط

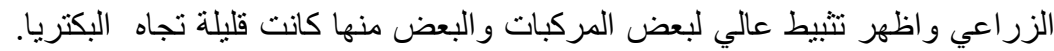

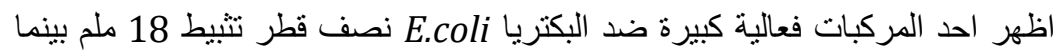
المركب الاخر أظهر فعالية ضد البكتيريا E.coli, S. aureues انصاف اقطار تثبيط

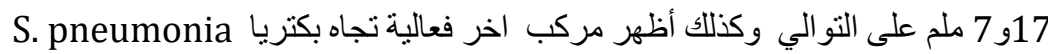

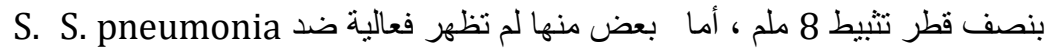

pyogenes ,
معلومات البحث:

تأريخ الاستلام: 2020/11/19 تأريخ القبـــول: 2021/01/09 تاريخ الام: 2020/11/19

الكلمات المفتاحية:

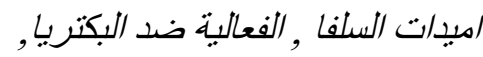
المركبات الحلقية غبير المتجانسة العالية

تشكل المركبات الحلقية غير المتجانسة أكبر مجموعة من المركبات العضوية وأكثرها تنوعًا. يوجد اليوم الكثير من المركبات

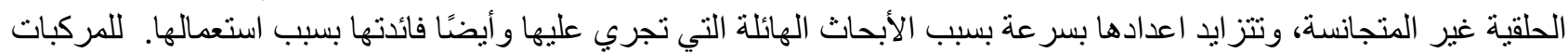

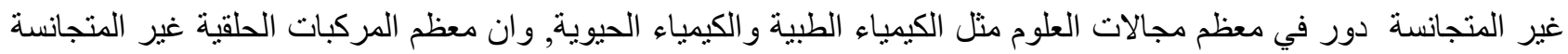

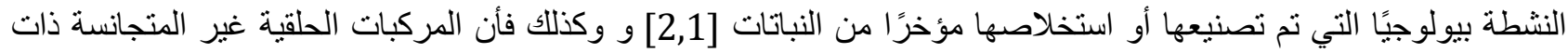

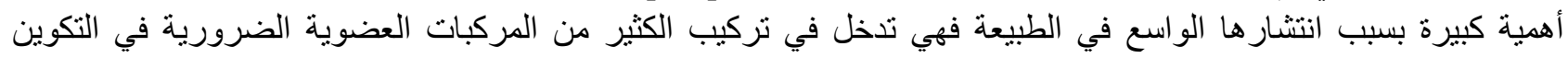

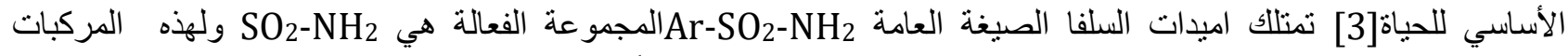

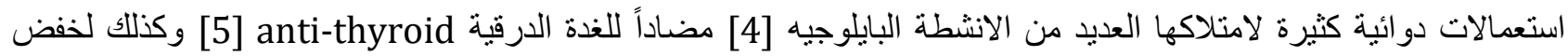

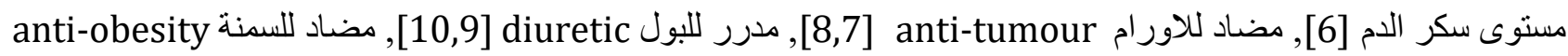

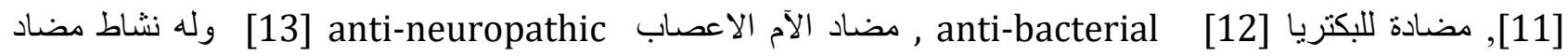

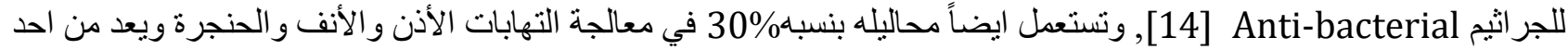

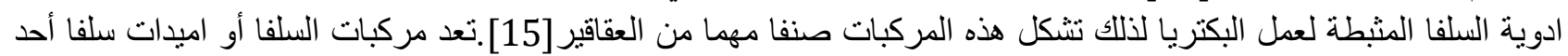

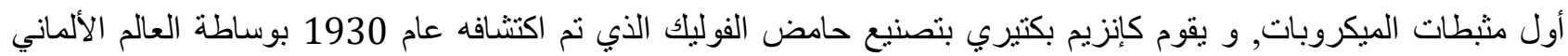
Domajk في الجسم ويصبح نشطا داخل الجسم, وأن اغلب مركبات Sulfonamides مشتقه من Sulfanilamide ويشابهه benzoic acid الاختلافات في المجموعة الوظيفية (RSO2NH2) إلى ظهور خصائص فسيولوجية وكيميائية ودوائية مختلفة لهذه الأدوية, 


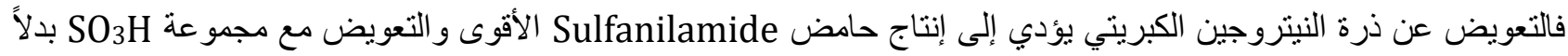

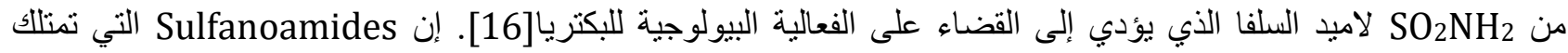

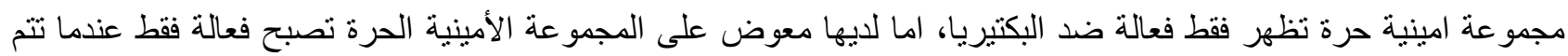

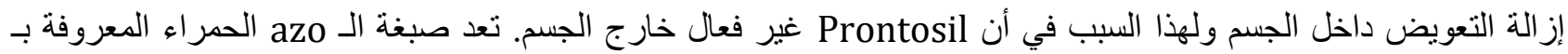
من اوائل Sulfonamides التي حددها Domagk و اخرون كما في المخطط 1 ، وان Prontosil

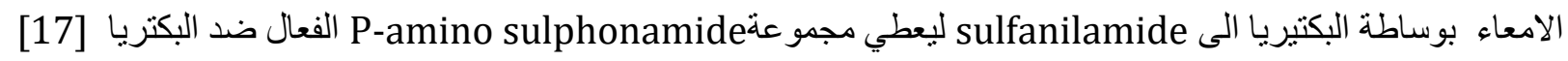<smiles>Nc1ccc(N=Nc2ccc(S(N)(=O)=O)cc2)c(N)c1</smiles>
المخطط 1: تحلل Prontosil Pي الامعاء.

$$
\text { تحضير المركبات (الموائق العمل }
$$

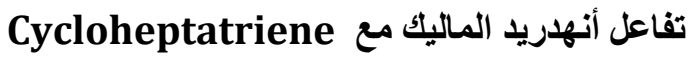

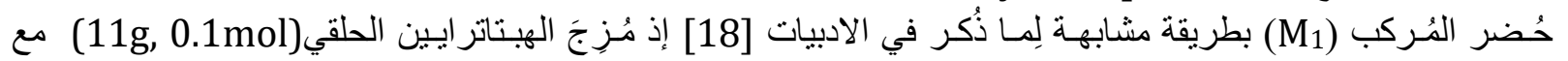

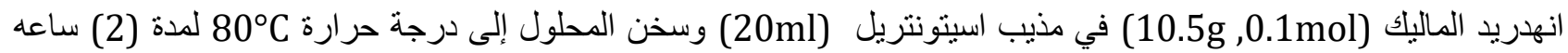

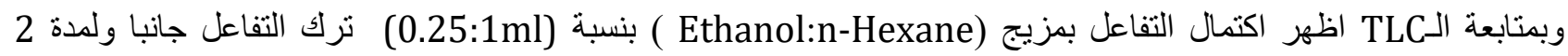

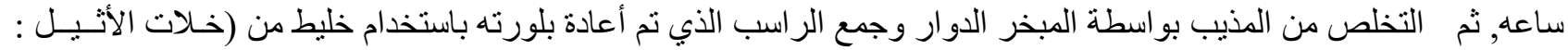

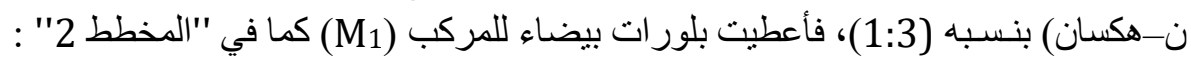

(4R,4aR,5aS,6S)-3-methylene-3,3a,4,4a,5,5a,6,6a-octahydro-1H-4,6ethenocyclopropa[f]isobenzofuran-1-one(M1)

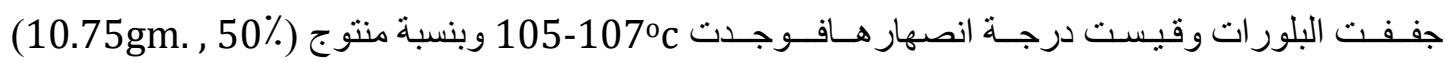
Umax (KBr): $2950 \mathrm{~cm}^{-1}$ (CH- aliph.) ; $1774 \mathrm{~cm}^{-1}$ (C=0) ; $1436 \mathrm{~cm}^{-1}$ (C=C);1224.71 cm-1(CO-O) .

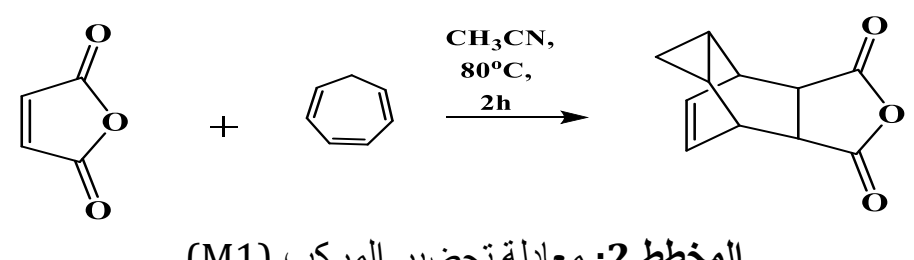

(المخطط 2: معادلة تحضير المركب (M1)

4'-amino-[1,1'-biphenyl]-4-aminium تفاعل M1 Mع

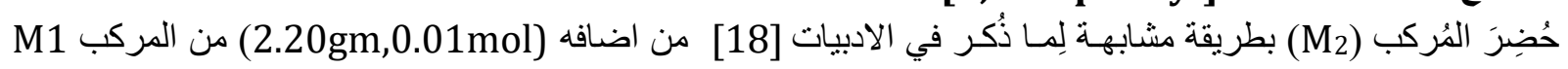

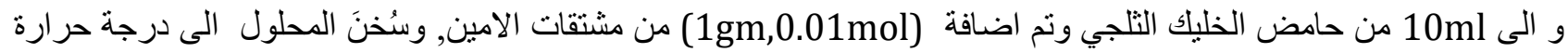
و 60C لمدة 3 ساعات وبمتابعة الـ TLC بمزيج من الايثانول: خلات الاثيل بنسبة (0.25:1m) اظظهر اكتمال التفاعل برد المزيج

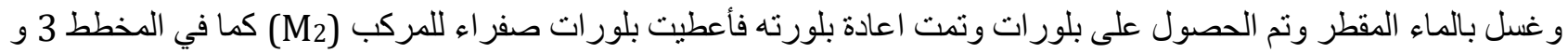
الجدول 1.

\section{4-(R,4aR,5aS,6S)-2-(4'-amino-[1,1'-biphenyl]-4-yl)4,4a,5,5a,6,6a-hexahydro-4,6-} ethenocyclopropa[f]isoindole-1,3 (2H,3aH)-dione $\left(\mathrm{M}_{3}\right)$

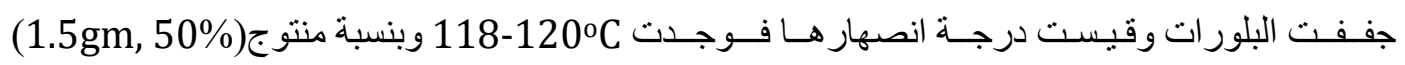


Umax (KBr ): $3008 \mathrm{~cm}^{-1}$ (CH-aromat.) ; $2952 \mathrm{~cm}^{-1}$ (CH- aliph.); $1658 \mathrm{~cm}^{-1}$ (C=0); $1500 \mathrm{~cm}^{-1}$ $(\mathrm{C}=\mathrm{C}) ; 1226 \mathrm{~cm}^{-1}(\mathrm{C}-\mathrm{N}) ; 3436,3352 \mathrm{~cm}^{-1}\left(\mathrm{NH}_{2}\right)$
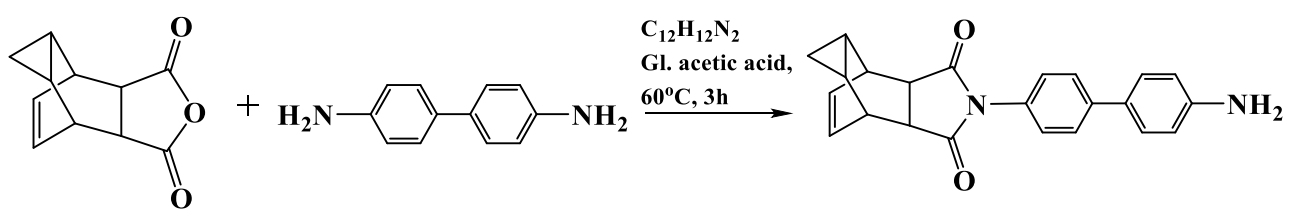

(المخطط :3 معادلة تحضير المركب(M2)

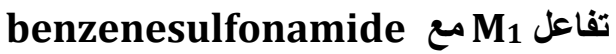

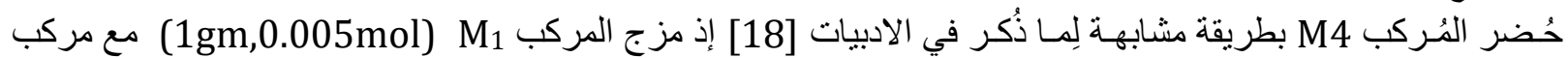

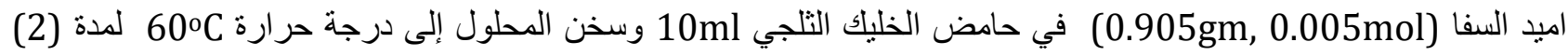

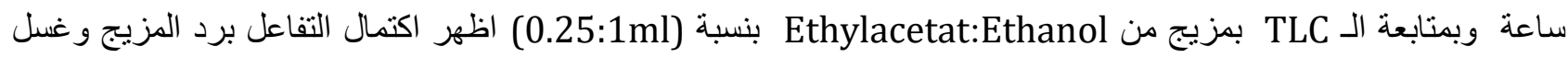

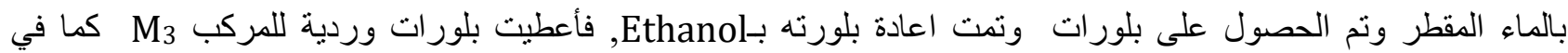

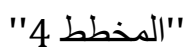

\section{4-((4R,4aR,5aS,6S)-1,3-dioxo-3,3a,4,4a,5,5a,6,6a-octahydro-4,6- ethenocyclopropa[f]isoindol-2(1H)-yl)benzenesulfonamide(M4)}

Pink crystals, m.p., 170-171ㅇ C ; (Yield: 1.5gm, 78\%); 3097 $\mathrm{cm}^{-1}$ (CH-aromat.) ; $2945 \mathrm{~cm}^{-1}$ (CH- aliph.); $1770 \mathrm{~cm}^{-1} \quad(\mathrm{C}=0) ; 1492 \mathrm{~cm}^{-1}(\mathrm{C}=\mathrm{C}) ; 1294 \mathrm{~cm}^{-1}(\mathrm{C}-\mathrm{N}) ; 3409,3288 \mathrm{~cm}^{-1}\left(\mathrm{NH}_{2}\right) ; 1168$, $1344 \mathrm{~cm}^{-1}(\mathrm{~S}=0) ; 609 \mathrm{~cm}^{-1}(\mathrm{~S}=0) ;{ }^{1} \mathrm{H}-\mathrm{NMR}\left(\mathrm{DMSO}-\mathrm{d}_{6}\right): \delta 6.85 \mathrm{ppm}\left(\mathrm{N}-\mathrm{H}_{2}, \mathrm{~s}\right) ; \delta 7.93-7.47(4 \mathrm{H}, \mathrm{dd}$, aromatic) ; $\delta 2.53 \mathrm{ppm}\left(2 \mathrm{H}, \mathrm{s}, \mathrm{H}_{4,8}\right) ; \delta 1.20 \mathrm{ppm}\left(2 \mathrm{H}, \mathrm{s}, \mathrm{H}_{5}, 7\right) ; \delta 0.28: 0.10 \mathrm{ppm}\left(2 \mathrm{H}, \mathrm{s}, \mathrm{H}_{6}\right) ; \delta 5.80$ $\operatorname{ppm}(2 \mathrm{H}, \mathrm{s}, \mathrm{H} 9,10) ; \delta 3.41 \mathrm{ppm}\left(4 \mathrm{H}, \mathrm{s}, \mathrm{H}_{11}, 12\right) \cdot{ }^{13} \mathrm{C}-\mathrm{NMR}$ (DMSO-d6): $\delta 145.12 \mathrm{ppm}\left(\mathrm{C}_{1}\right.$, aromatic) ; $\delta$ 128.03-122.03ppm ( $\left(\mathrm{C}_{2,2-3,3-}\right) ; \delta 144.12\left(\mathrm{C}_{4}\right.$, aromatic $) ; \delta 177.65\left(\mathrm{C}_{5,15}\right.$ carbonel $) ; \delta$ $45.48\left(\mathrm{C}_{6,10}\right) ; \delta$ 9.88ppm $\left(\mathrm{C}_{7,9}\right) ; \delta 9.91 \mathrm{ppm}\left(\mathrm{C}_{8}\right) ; \delta 135.38 \mathrm{ppm}\left(\mathrm{C}_{11,12}\right) ; \delta 40.47 \mathrm{ppm}\left(\mathrm{C}_{13,14}\right)$
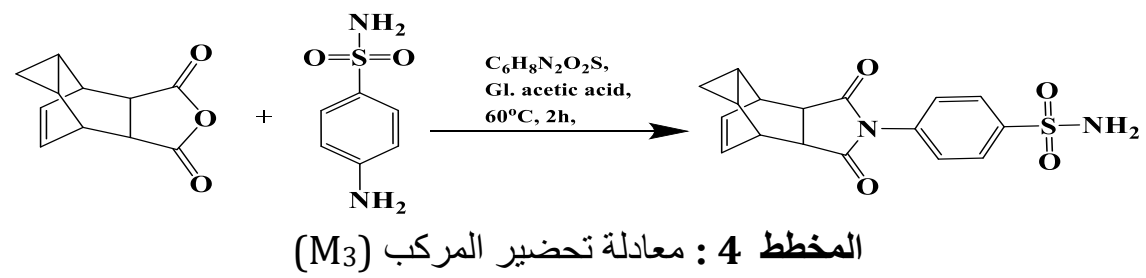

4-(methylbenzenesulfonyl chloride) تفاعل M

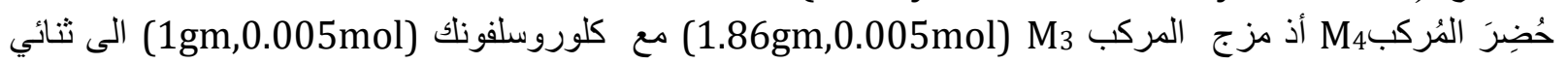

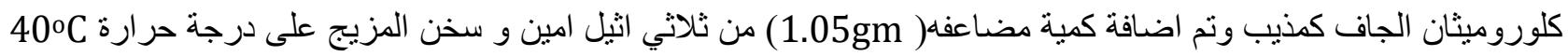

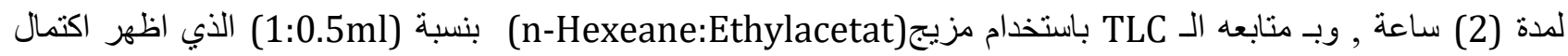

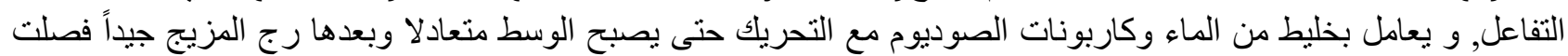

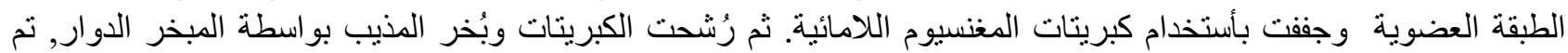

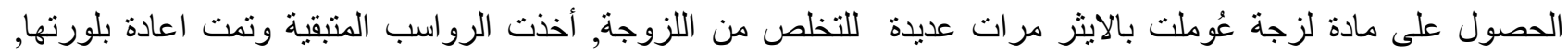

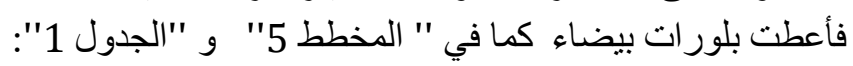

$N-(4 '-((4 R, 4 a R, 5 a S, 6 S)-1,3-d i o x o-3,3 a, 4,4 a, 5,5 a, 6,6 a-o c t a h y d r o-4,6-$ ethenocyclopropa[f]isoindol-2(1H)-yl)-[1,1'-biphenyl]-4-yl)-4-methyl benzenesulfonamide( $\left(\mathrm{M}_{4}\right)$

Beige crystals, m.p., 230-231 ${ }^{\circ} \mathrm{C}$; (Yield: $\left.1.5 \mathrm{~g}, 52 \%\right)$; $\mathrm{U}_{\max }(\mathrm{KBr}): 3008 \mathrm{~cm}^{-1}$ (CH-aromat.); $2954 \mathrm{~cm}^{-1} \quad$ (CH- aliph.); $1670 \mathrm{~cm}^{-1} \quad(\mathrm{C}=0) ; 1556 \mathrm{~cm}^{-1} \quad(\mathrm{C}=\mathrm{C}) ; 1089 \mathrm{~cm}^{-1}(\mathrm{C}-\mathrm{N}) ; 3452,3370 \mathrm{~cm}^{-1}$ $\left(\mathrm{NH}_{2}\right) ; 1174,1375 \mathrm{~cm}^{-1}(\mathrm{~S}=0) ; 594 \mathrm{~cm}^{-1}(\mathrm{~S}=0) ;{ }^{1} \mathrm{H}-\mathrm{NMR}$ (DMSO-d6): $\delta 9.11 \mathrm{ppm}(\mathrm{N}-\mathrm{H}, \mathrm{s}) ; \delta$ $2.45 \mathrm{ppm}\left(\mathrm{C}-\mathrm{H}_{3}, \mathrm{~s}\right) ; \delta 7.76-7.18(4 \mathrm{H}, \mathrm{dd}$, aromatic $) ; \delta 7.11-7.09(4 \mathrm{H}, \mathrm{dd}$, aromatic $) ; \delta 7.21-$ 
$7.66(4 \mathrm{H}, \mathrm{dd}$, aromatic $) ; \delta 2.29 \mathrm{ppm}(2 \mathrm{H}, \mathrm{m}, \mathrm{H} 9,13) ; \delta 1.23 \mathrm{ppm}\left(2 \mathrm{H}, \mathrm{m}, \mathrm{H}_{10,12}\right) ; \delta$ 0.28:0.07ppm $\left(2 \mathrm{H}, \mathrm{m}, \mathrm{H}_{11}\right) ; \delta 5.70 \mathrm{ppm}\left(2 \mathrm{H}, \mathrm{s}, \mathrm{H}_{14,15}\right) ; \delta 3.37 \mathrm{ppm}\left(4 \mathrm{H}, \mathrm{m}, \mathrm{H}_{16,17}\right) .{ }^{13} \mathrm{C}-\mathrm{NMR}$ (DMSO-d 6$) ; \delta 33.75$

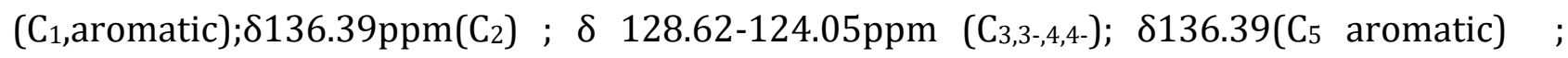
$\delta 138.39\left(\mathrm{C}_{6} \quad\right.$ aromatic $) \quad ; \quad \delta \quad 126.04-113.04 \mathrm{ppm} \quad\left(\mathrm{C}_{7,7-8,8--}\right) ; \quad \delta 141.39\left(\mathrm{C}_{9}\right.$, aromatic); $\delta 141.39\left(\mathrm{C}_{10, \text { aromatic }) ; ~} \delta 128.71-124.75 \mathrm{ppm}\left(\mathrm{C}_{11,12}\right) ; \delta 136.39\left(\mathrm{C}_{13}\right.\right.$ aromatic $)$; $\delta 176.65\left(\mathrm{C}_{14,24}\right.$ Carbonel $) ; \delta 45.39\left(\mathrm{C}_{15,19}\right) ; \delta$ 9.93ppm $\left(\mathrm{C}_{16,18}\right) ; \delta 9.87 \mathrm{ppm}\left(\mathrm{C}_{17}\right) ; \delta 133.38 \mathrm{ppm}$ $\left(\mathrm{C}_{20,21)} ; \delta 42.50 \mathrm{ppm}\left(\mathrm{C}_{22,23}\right)\right.$

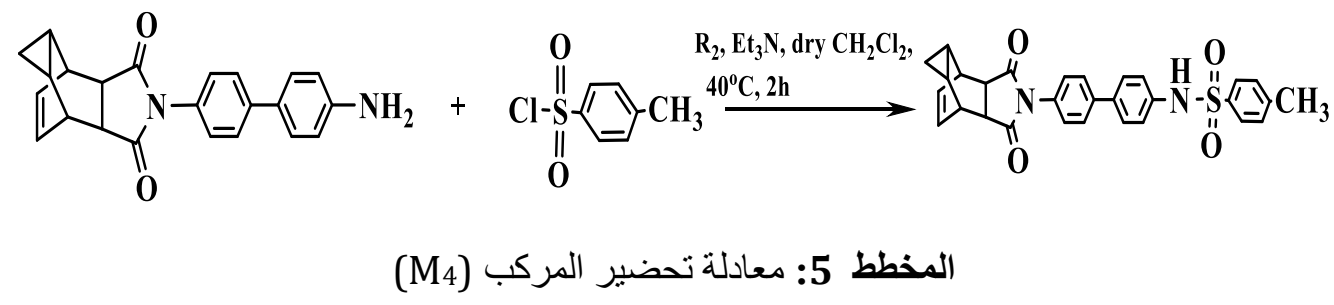

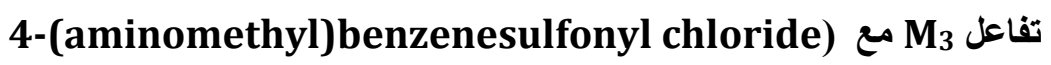

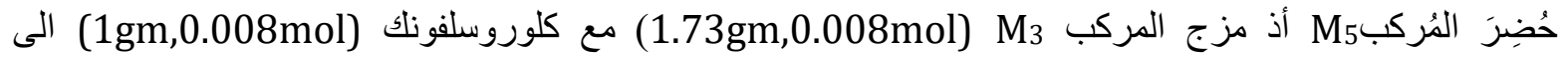

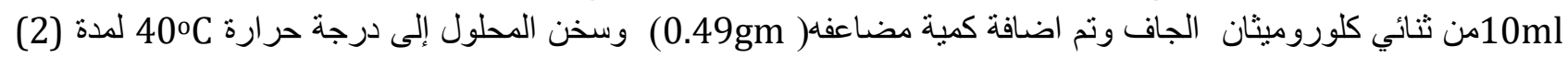

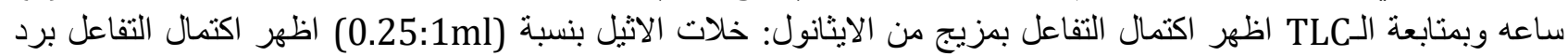

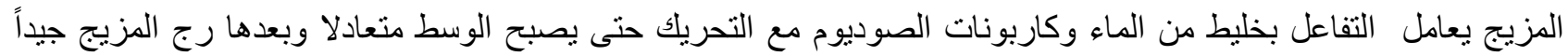

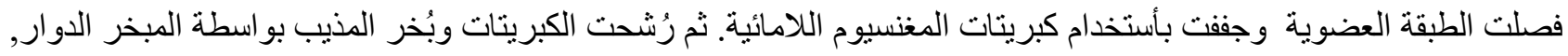

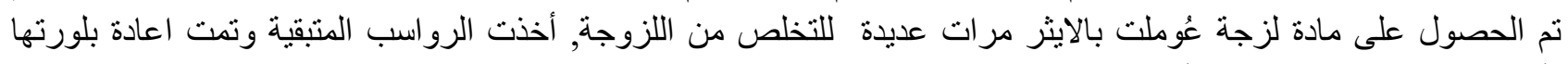

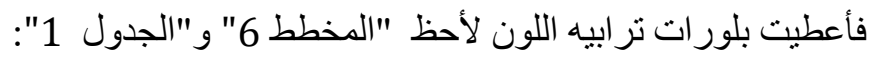

\section{4-(( $\lambda^{4}$ azaneyl)methyl)-N-(4'-((4R,4aR,5aS,6S)-1,3-dioxo-3,3a,4,4a, 5,5a,6,6a-octahydro-} 4,6-ethenocyclopropa[f]isoindol-2(1H)-yl)-[1,1'-biphenyl]-4-yl)benzenesulfonamide(M5)

White crystals, m.p., 250-254ㅇ C ; (Yield: $1.6 \mathrm{~g}, 60 \%)$; $\mathrm{U}_{\max }\left(\mathrm{KBr}\right.$ ): $3056 \mathrm{~cm}^{-1}$ (CH-aromat.); $2970 \mathrm{~cm}^{-1} \quad$ (CH- aliph.); $1660 \mathrm{~cm}^{-1} \quad(\mathrm{C}=0) ; 1550 \mathrm{~cm}^{-1} \quad(\mathrm{C}=\mathrm{C}) ; 1317 \mathrm{~cm}^{-1}(\mathrm{C}-\mathrm{N}) ; 3344 \mathrm{~cm}^{-1}\left(\mathrm{NH}_{2}\right)$; $1172,1375 \mathrm{~cm}^{-1}(\mathrm{~S}=0) ; 543 \mathrm{~cm}^{-1}(\mathrm{~S}=0) ;{ }^{1} \mathrm{H}-\mathrm{NMR}$ (DMSO-d $): \delta 9.85 \mathrm{ppm}(\mathrm{N}-\mathrm{H}, \mathrm{s}) ; \delta 8.12 \mathrm{ppm}$ $\left(\mathrm{N}-\mathrm{H}_{2}, \mathrm{~s}\right) ; \delta 4.01 \mathrm{ppm}\left(\mathrm{C}-\mathrm{H}_{2}, \mathrm{~s}\right) ; \delta 7.61-7.36(4 \mathrm{H}, \mathrm{dd}$, aromatic) $; \delta 7.10-6.99(4 \mathrm{H}, \mathrm{dd}$, aromatic) ; $\delta$ 7.76-7.20(4H, dd, aromatic) ; $\delta 2.49 \mathrm{ppm}\left(2 \mathrm{H}, \mathrm{m}, \mathrm{H}_{10}, 14\right) ; \delta 1.29 \mathrm{ppm}\left(2 \mathrm{H}, \mathrm{m}, \mathrm{H}_{11}, 13\right) ; \delta$ 0.28:0.03 ppm ( $\left.2 \mathrm{H}, \mathrm{m}, \mathrm{H}_{12}\right) ; \delta 5.83 \mathrm{ppm}\left(2 \mathrm{H}, \mathrm{m}, \mathrm{H}_{15}, 16\right) ; \delta 3.45 \mathrm{ppm}\left(4 \mathrm{H}, \mathrm{m}, \mathrm{H}_{17}, 18\right)$. ${ }^{13} \mathrm{C}-\mathrm{NMR}$ (DMSO-d $) ; \quad \delta 45.39\left(\mathrm{C}_{1}\right.$,aromatic); $\delta 148.39 \mathrm{ppm}\left(\mathrm{C}_{2}\right) \quad ; \quad \delta \quad 124.75-124.05 \mathrm{ppm} \quad\left(\mathrm{C}_{3,3-4,4-}\right)$;

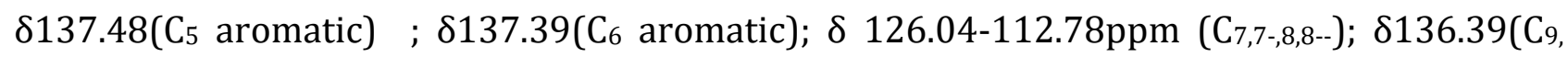
aromatic); $\delta 136.39\left(\mathrm{C}_{10}\right.$, aromatic); $\delta 126.04-124.75 \mathrm{ppm}\left(\mathrm{C}_{11,12}\right) ; \delta 128.71\left(\mathrm{C}_{13}\right.$ aromatic $)$; $\delta 177.65$ ( $\mathrm{C}_{14,24}$ Carbonel); $\delta 42.50\left(\mathrm{C}_{15,19}\right) ; \delta 9.93 p p m\left(\mathrm{C}_{16,18}\right) ; \delta 9.64 p p m\left(\mathrm{C}_{17}\right) ; \delta 128.62 \mathrm{ppm}$ $\left(\mathrm{C}_{20,21)}\right.$ $\delta 40.45 \mathrm{ppm}\left(\mathrm{C}_{22,23)}\right.$

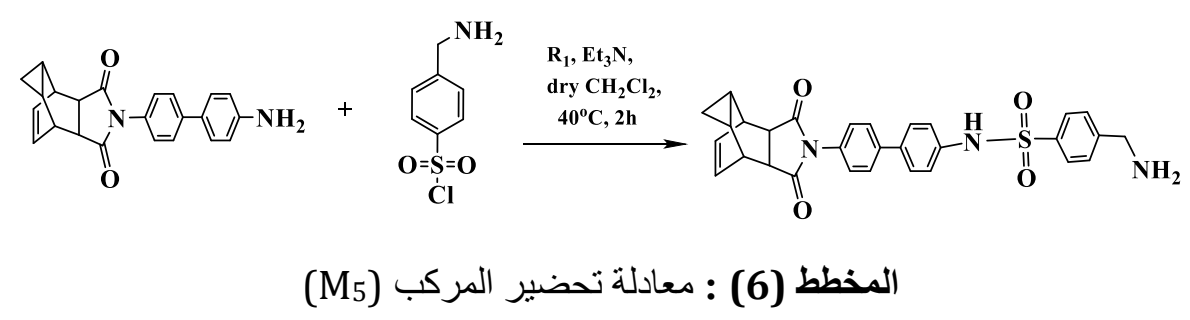

\footnotetext{
طرق تحضير الاطباق والبكتريا

تحضير وسط أجار مولر-هينتون: Prepare Mueller-Hinton agar medium
} 
تم تحضير Mueller-Hinton Agar وفقًا لتعليمات الشركة الصانعة عن طريق إضافة 38 جم من مسحوق أجار مغذي وجاف إلى لتر من الماء المقطر في دورق مخروطي، ويتم تسخين الخليط حتى يذوب، ثم يغلق فوهة القارورة بالقطن، وتعقيم

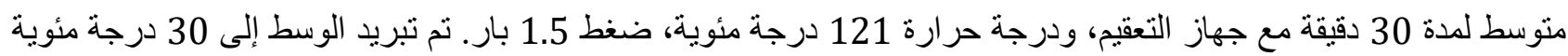

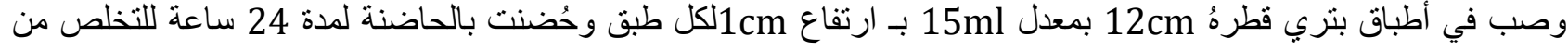
بخار الماء ولمعرفة مدى سلامة الاكار من التلوث، ثم وضعت بالثلاجة 24 ساعة لغرض التصلب التام للأكار، ثم جرى أثى استخدامها بعد ذلك.

\section{Prepare bacteria}

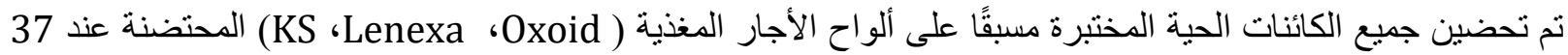
درجة مئوية لمدة 24 ساعة ، وتم تحضير المعلقات البكتيرية لكل من العزلات النية النقية ومصدرها(مصدر العزلات البات البكتريا السالبة

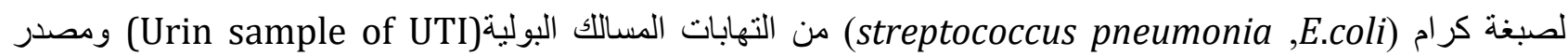
العزلات البكتريا الموجبة لصبغة الكرام (Streptococcus pyogenes, staphyllococcus aerus) من التهابات

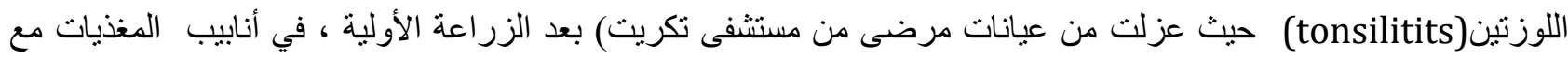
McFarland 0.6 العقدية الرئوية، والبكتيريا إيجابية الجرام العقدية المقيحة، المكور ات العنية العنقودية الذهبية.

\section{تقنية نشر واختبار تقدير الحد الأدنى للتركيز المثبط}

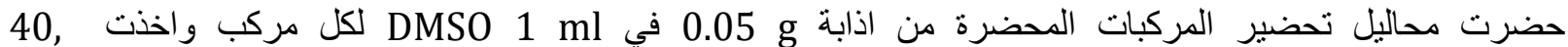

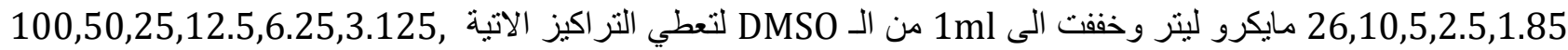

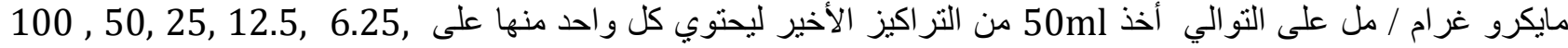

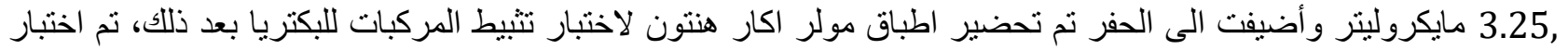

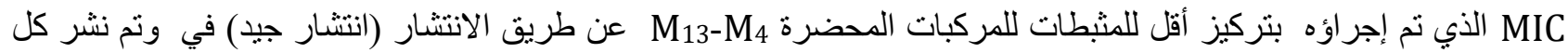

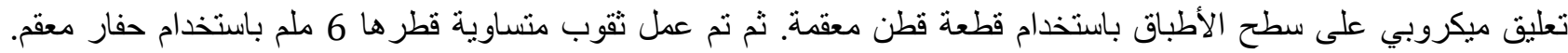

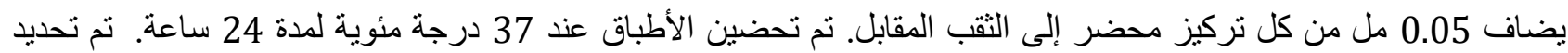
النشاط المضاد للبكتيريا عن طريق قياس قطر منطقة التنبيط حول كل ثقب في ملم. وقورنت تللك المركبات مع المضادات الحيوية Azithromycin 15mcg و Cephalosporin 30mcg, Amoxicillin 20mcg, Ampicillin,25mcg . [19] Ciprofloxacin 30mcg و Doxycycline 30mcg g

النتائج والمناقشة

تم تشخيص المركبات المحضرة M5-M1 وطيف الرنين النووي المغناطيسي للبروتون وتم التأكد من حصول التفاعل من خلال ملاحظة التغيرات التي حصلت على الصفات الفيزيائية من درجة الانصهار و التغير الكبير التير التيات

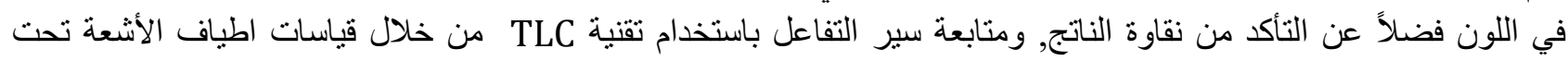
الحمر اء IR ، وان تقنية IR مهمة جداً في التشخيص العضوي للمركبات المحضرة في الحالثين السائلة والصلبة, حيث توفر هذه

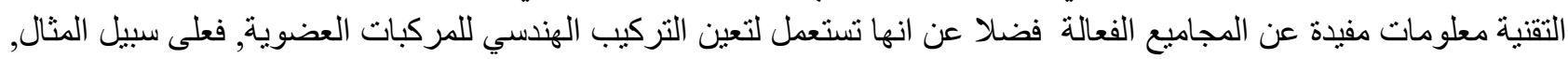

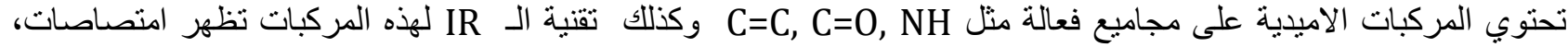

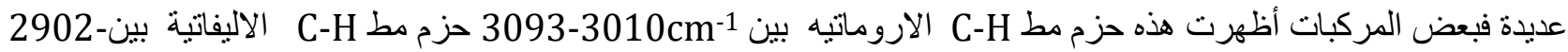

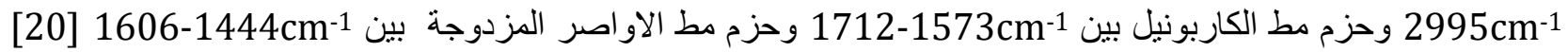

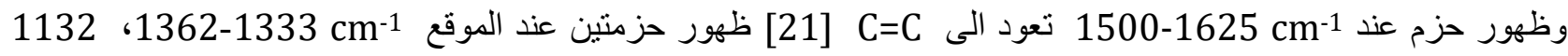

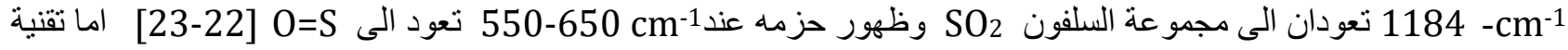
اطياف 1H-NMR حيت تعد هذه التقنية مهمة جدا في التشخيص العضوي للمركبات المحضرة في الحالة السائلة، أذ نوفر هذه

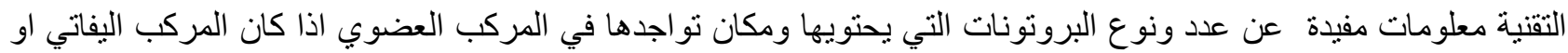

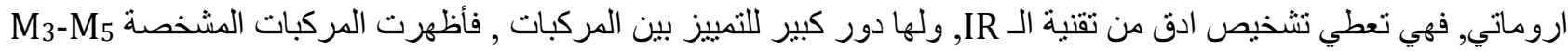

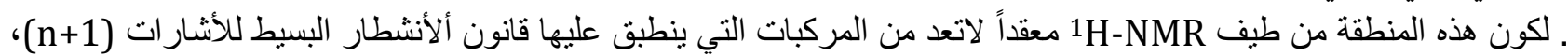

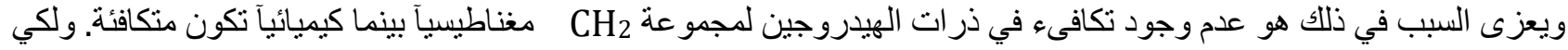

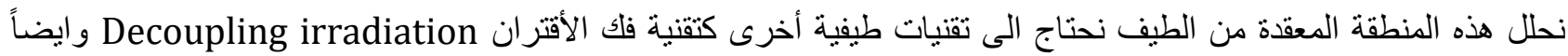

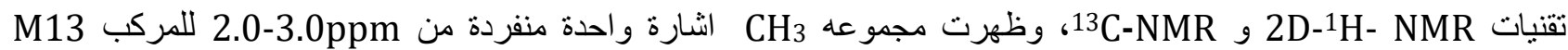


4.5-6.5ppm وظهرت

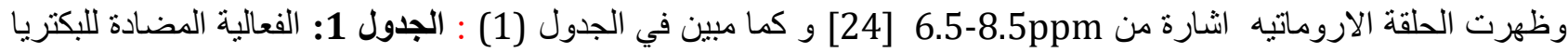
للمركبات المحضرة لنمو عدد من الجر اثثم السالبة و الموجبة (قطر دائرة الثرة التثبيط مقاسة بـ ملم).

\begin{tabular}{|c|c|c|c|c|c|}
\hline $\begin{array}{c}\text { Compd } \\
\text { No. }\end{array}$ & Compd. Structure of & $\begin{array}{c}\text { Chemical } \\
\text { shift } \\
\delta) p p m(\end{array}$ & $\begin{array}{c}\text { No of } \\
\text { Carbon }\end{array}$ & $\mathbf{T}$ & $\begin{array}{c}\text { Type } \\
\text { of proton }\end{array}$ \\
\hline $\mathrm{M}_{4}$ & $\mathbb{\|}$ & $\begin{array}{c}3.41 \\
5.80 \\
0.28: 0.10 \\
1.20 \\
2.53 \\
7.93-7.47 \\
6.85\end{array}$ & $\begin{array}{l}\mathrm{m} \\
\mathrm{m} \\
\mathrm{m} \\
\mathrm{m} \\
\mathrm{m} \\
\mathrm{dd} \\
\mathrm{S}\end{array}$ & $\begin{array}{l}2 \mathrm{H} \\
2 \mathrm{H} \\
2 \mathrm{H} \\
2 \mathrm{H} \\
2 \mathrm{H} \\
4 \mathrm{H} \\
2 \mathrm{H}\end{array}$ & $\begin{array}{c}\mathrm{H}_{11,12} \\
\mathrm{H}_{9,10} \\
\mathrm{H}_{6} \\
\mathrm{H}_{5,7} \\
\mathrm{H}_{4,8} \\
\mathrm{H}_{2,2-3,3-} \\
\mathrm{H}_{1}\end{array}$ \\
\hline $\mathrm{M}_{12}$ & & $\begin{array}{c}3.37 \\
5.70 \\
0.28: 0.07 \\
1.23 \\
2.29 \\
7.21-7.66 \\
7.11-7.09 \\
7.76-7.18 \\
9.11 \\
2.45\end{array}$ & $\begin{array}{l}\mathrm{m} \\
\mathrm{m} \\
\mathrm{m} \\
\mathrm{m} \\
\mathrm{m} \\
\mathrm{dd} \\
\mathrm{dd} \\
\mathrm{dd} \\
\mathrm{m} \\
\mathrm{S}\end{array}$ & $\begin{array}{l}2 \mathrm{H} \\
2 \mathrm{H} \\
2 \mathrm{H} \\
2 \mathrm{H} \\
2 \mathrm{H} \\
4 \mathrm{H} \\
4 \mathrm{H} \\
4 \mathrm{H} \\
1 \mathrm{H} \\
3 \mathrm{H}\end{array}$ & $\begin{array}{c}\mathrm{H}_{16,17} \\
\mathrm{H}_{14,15} \\
\mathrm{H}_{11} \\
\mathrm{H}_{10,12} \\
\mathrm{H}_{9,13} \\
\mathrm{H}_{7,7-, 8,8-} \\
\mathrm{H}_{5,5-, 6,6-} \\
\mathrm{H}_{3,3-, 4,4-} \\
\mathrm{H}_{2} \\
\mathrm{CH}_{3}\end{array}$ \\
\hline $\mathrm{M}_{13}$ & & $\begin{array}{c}3.45 \\
5.83 \\
0.28: 0.03 \\
1.29 \\
2.49 \\
7.61-7.36 \\
7.10-6.99 \\
7.76-7.20 \\
4.01 \\
9.85 \\
8.12\end{array}$ & $\begin{array}{c}\mathrm{m} \\
\mathrm{m} \\
\mathrm{m} \\
\mathrm{m} \\
\mathrm{m} \\
\mathrm{dd} \\
\mathrm{dd} \\
\mathrm{dd} \\
\mathrm{S} \\
\mathrm{S} \\
\mathrm{S}\end{array}$ & $\begin{array}{l}2 \mathrm{H} \\
2 \mathrm{H} \\
2 \mathrm{H} \\
2 \mathrm{H} \\
2 \mathrm{H} \\
4 \mathrm{H} \\
4 \mathrm{H} \\
4 \mathrm{H} \\
2 \mathrm{H} \\
1 \mathrm{H} \\
3 \mathrm{H}\end{array}$ & $\begin{array}{c}\mathrm{H}_{17,18} \\
\mathrm{H}_{15,16} \\
\mathrm{H}_{12} \\
\mathrm{H}_{11,13} \\
\mathrm{H}_{10,14} \\
\mathrm{H}_{8,8-}, 9,9- \\
\mathrm{H}_{6,6-, 7,7-} \\
\mathrm{H}_{4,4-5,5-} \\
\mathrm{H}_{3} \\
\mathrm{H}_{2} \\
\mathrm{H}_{1}\end{array}$ \\
\hline
\end{tabular}




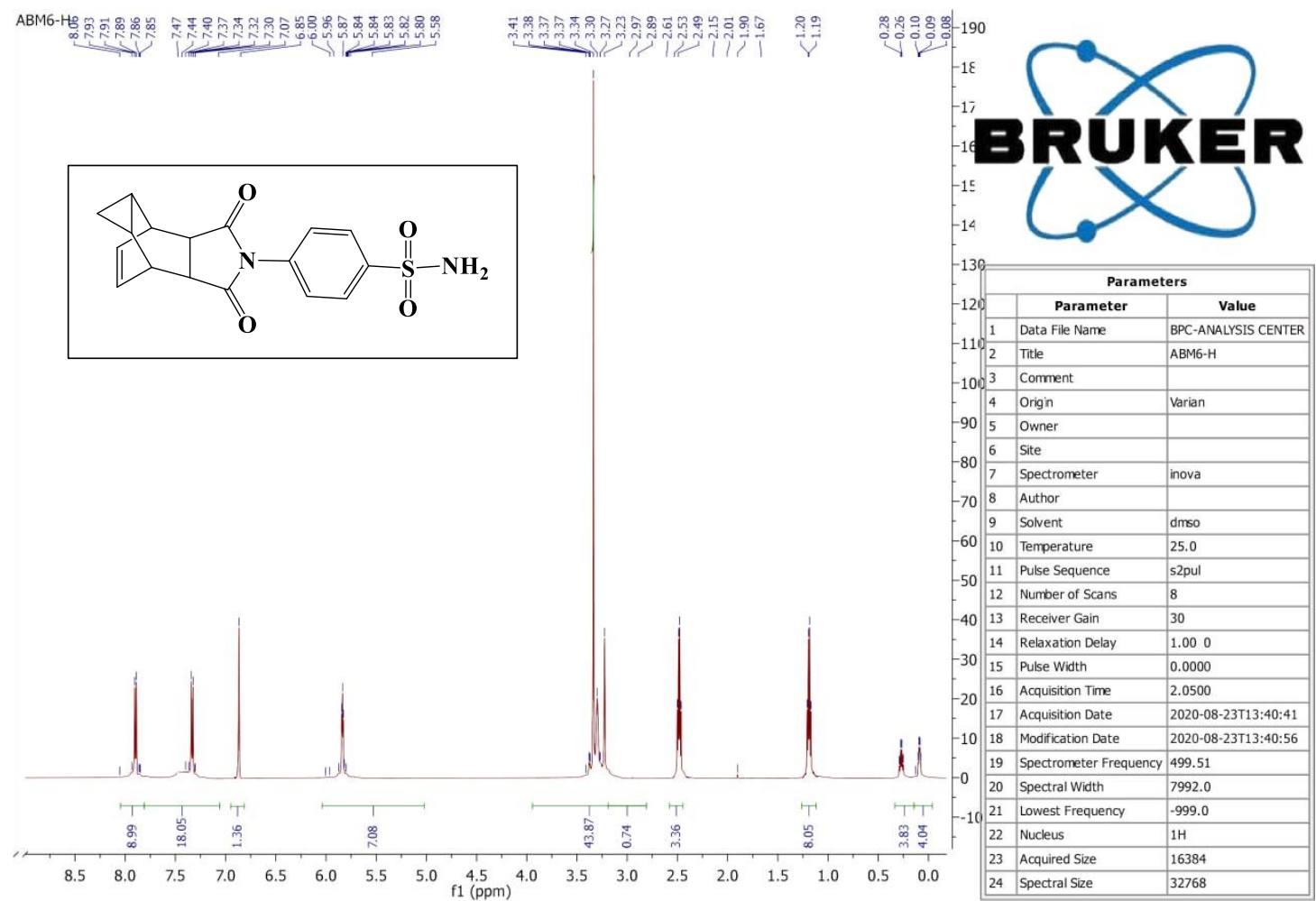

H-NMR شكل(1) طيف الرنين النووي المغناطيسي

ABM日十

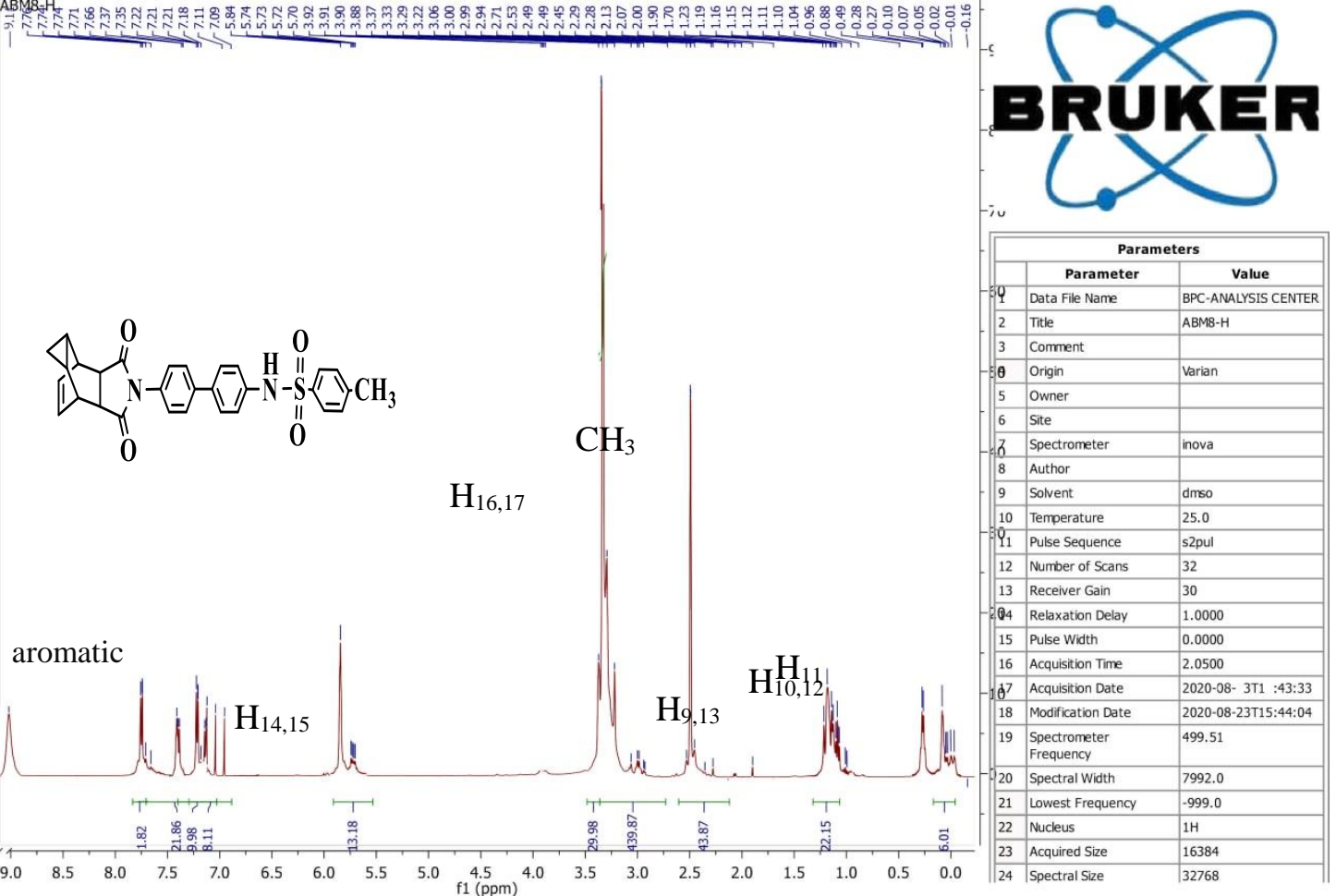

$\mathrm{NH}$

Mكل (2) طيف الرنين النووي المغناطيسي H-NMR للمركب 


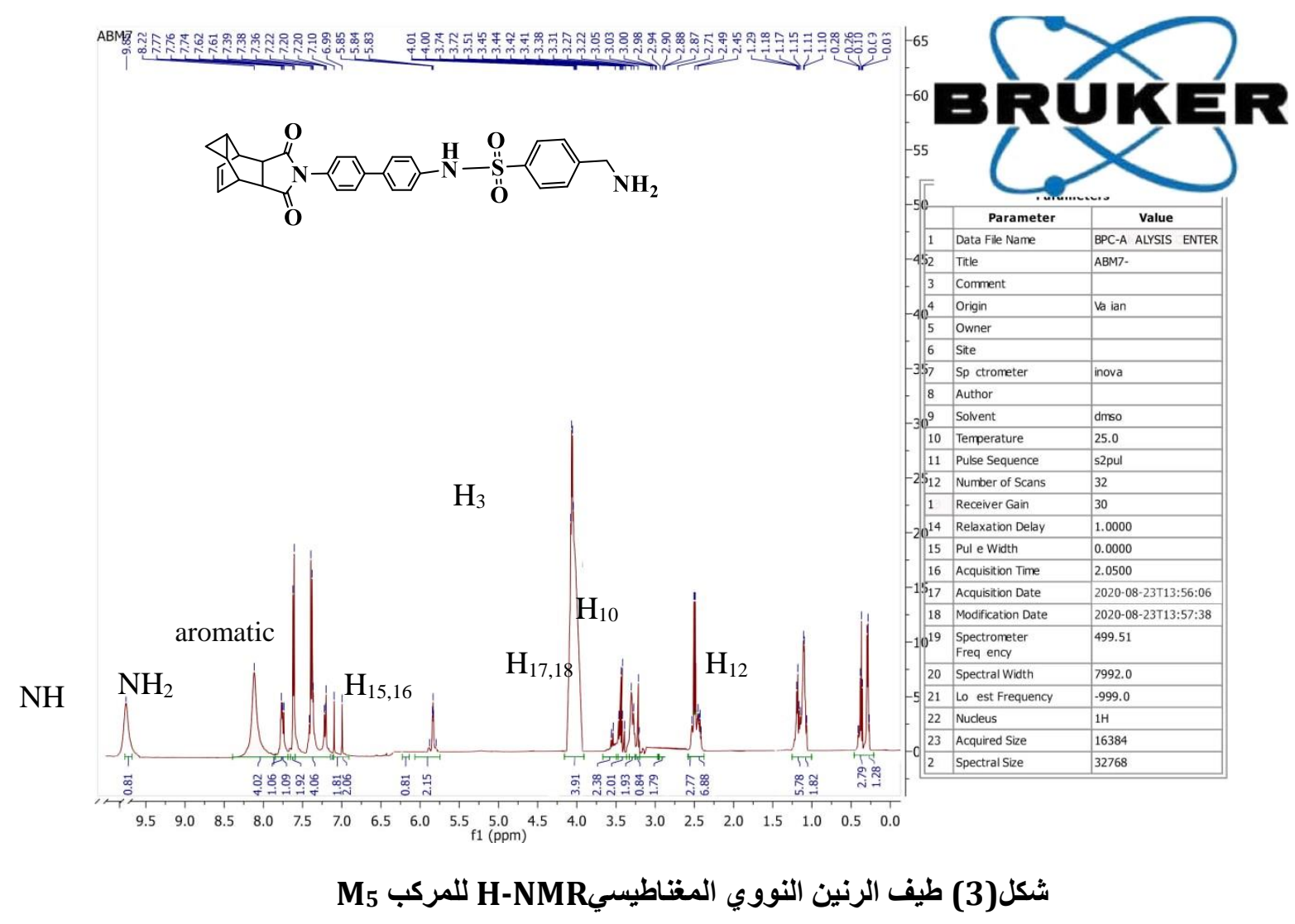

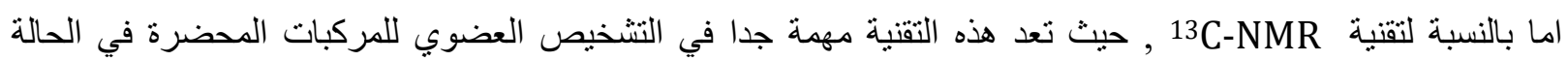
السائلة, حيث توفر هذه التقنية معلومات مفيدة عن عدد ذرات الكاربون التي يحتويها ومكان تو اجدها في المركبات العضوية

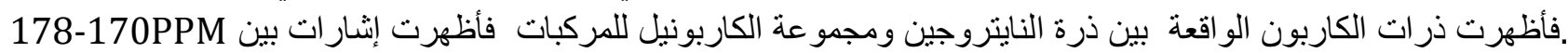

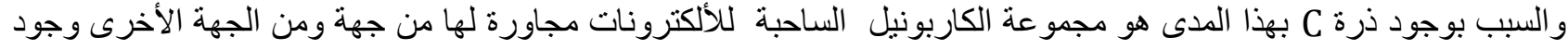

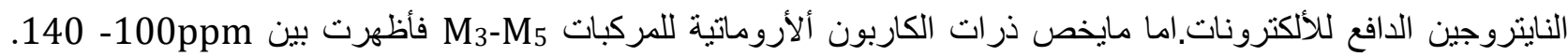

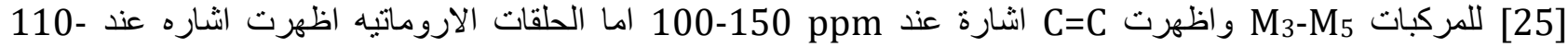

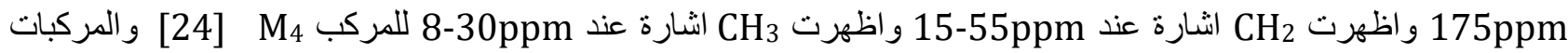
المحضرة كما مبين بالجدول (2 ) وأشكال أطياف 175-NMR للمركبات المحضرة (M3-M5).

\begin{tabular}{|c|c|c|c|c|}
\hline $\begin{array}{l}\text { Compd } \\
\text { No. }\end{array}$ & Compd. Structure of & 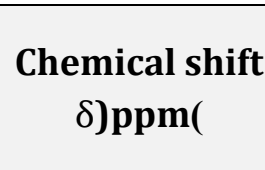 & $\begin{array}{c}\text { No. of } \\
\text { carbon }\end{array}$ & $\begin{array}{c}\text { Type } \\
\text { of } \\
\text { carbon }\end{array}$ \\
\hline $\mathrm{M}_{4}$ & 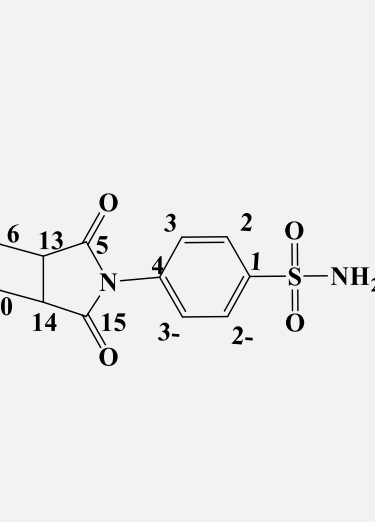 & $\begin{array}{c}40.47 \\
135.38 \\
9.91 \\
9.88 \\
45.48 \\
177.65 \\
144.12 \\
128.03-122.03 \\
145.12\end{array}$ & $\begin{array}{l}\mathrm{C}_{13,14} \\
\mathrm{C}_{11,12} \\
\mathrm{C}_{8} \\
\mathrm{C}_{7,9} \\
\mathrm{C}_{6,10} \\
\mathrm{C}_{5,15} \\
\mathrm{C}_{4} \\
\mathrm{C}_{2,2-3,3-} \\
\mathrm{C}_{1}\end{array}$ & $\begin{array}{l}\text { aliph } \\
\text { aliph } \\
\text { aliph } \\
\text { aliph } \\
\text { aliph } \\
\text { carbonel } \\
\text { aliph } \\
\text { aromatic } \\
\text { aromatic } \\
\text { aromatic }\end{array}$ \\
\hline
\end{tabular}




\begin{tabular}{|c|c|c|c|c|}
\hline $\mathrm{M}_{12}$ & 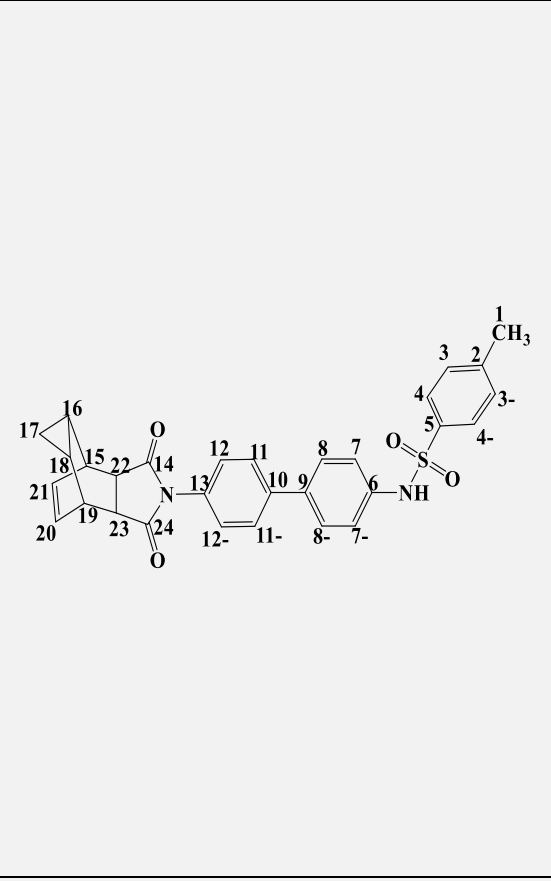 & $\begin{array}{c}42.50 \\
133.38 \\
9.87 \\
9.93 \\
45.39 \\
176.65 \\
136.39 \\
128.71-124.75 \\
141.93 \\
141.93 \\
126.04-113.04 \\
138.39 \\
136.93 \\
128.62-124.05 \\
136.39 \\
33.75\end{array}$ & $\begin{array}{l}\mathrm{C}_{22,23} \\
\mathrm{C}_{20,21} \\
\mathrm{C}_{17} \\
\mathrm{C}_{16,18} \\
\mathrm{C}_{15,19} \\
\mathrm{C}_{14,24} \\
\mathrm{C}_{13} \\
\mathrm{C}_{11,12} \\
\mathrm{C}_{10} \\
\mathrm{C}_{9} \\
\mathrm{C}_{7,7-8,8-} \\
\mathrm{C}_{6} \\
\mathrm{C}_{5} \\
\mathrm{C}_{3,3-4,4-} \\
\mathrm{C}_{2} \\
\mathrm{C}_{1}\end{array}$ & $\begin{array}{l}\text { aliph } \\
\text { aliph } \\
\text { aliph } \\
\text { aliph } \\
\text { aliph } \\
\text { carbonel } \\
\text { aliph } \\
\text { aromatic } \\
\text { aromatic } \\
\text { aromatic } \\
\text { aromatic } \\
\text { aromatic } \\
\text { aromatic } \\
\text { aromatic } \\
\text { aromatic } \\
\text { aromatic } \\
\text { aliphtic } \\
\end{array}$ \\
\hline $\mathrm{M}_{13}$ & (1) & $\begin{array}{c}40.45 \\
128.62 \\
9.64 \\
9.93 \\
42.50 \\
177.65 \\
128.71 \\
126.04-124.75 \\
136.39 \\
136.39 \\
126.04-112.78 \\
137.39 \\
137.48 \\
124.75-124.05 \\
148.93 \\
45.39\end{array}$ & $\begin{array}{l}\mathrm{C}_{22,23} \\
\mathrm{C}_{20,21} \\
\mathrm{C}_{17} \\
\mathrm{C}_{16,18} \\
\mathrm{C}_{15,19} \\
\mathrm{C}_{14,24} \\
\mathrm{C}_{13} \\
\mathrm{C}_{11,12} \\
\mathrm{C}_{10} \\
\mathrm{C}_{9} \\
\mathrm{C}_{7,7-8,8-} \\
\mathrm{C}_{6} \\
\mathrm{C}_{5} \\
\mathrm{C}_{3,3-4,4-} \\
\mathrm{C}_{2} \\
\mathrm{C}_{1}\end{array}$ & $\begin{array}{l}\text { aliph } \\
\text { aliph } \\
\text { aliph } \\
\text { aliph } \\
\text { aliph } \\
\text { carbonel } \\
\text { aliph } \\
\text { aromatic } \\
\text { aromatic } \\
\text { aromatic } \\
\text { aromatic } \\
\text { aromatic } \\
\text { aromatic } \\
\text { aromatic } \\
\text { aromatic } \\
\text { aromatic } \\
\text { aliphtic }\end{array}$ \\
\hline
\end{tabular}

وتم تقييم الفعالية البايولوجية لبعض المركبات اميدات السلفا المحضرة حيث تمتاز مركبات الحلقات غير المتجانسة

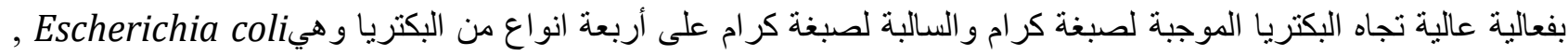
Streptococcus pyogenes, Staphylococcus aureus, Streptococcus pneumonia

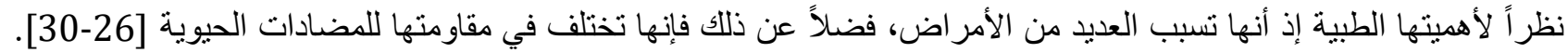
وتم استعمال المضادات الحيوية Amoxicillin ,Ampicillin ,Ciprofloxacin, Azithromycin , كعينات للسيطرة وكما في "الجدول 2" , وقد تم تقييم الفعالية البايولوجية لبعض المركبات المحضرة باستخدام طريقة الحفر

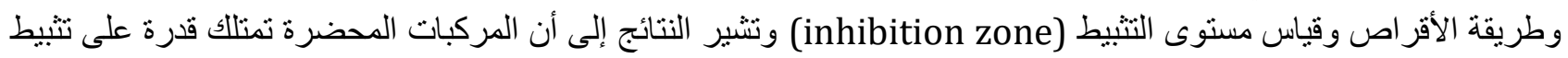

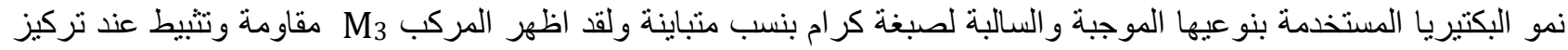

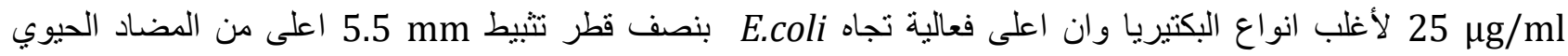

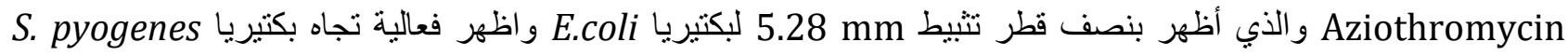

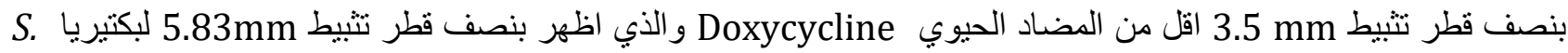

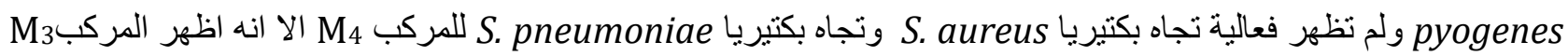

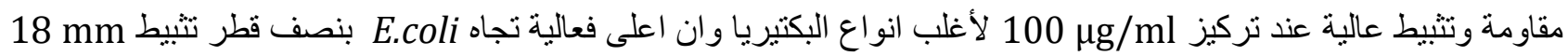


اعلى من المضاد الحيوي Ampicillin والذي أظهر اظهر بنصف قطر تثبيط 9.28 mm لبكتيريا E.coli و اظهر فعالية تجاه بكتيريا Syogenes بنصف قطر تنيط 7.5 mm اعلى من المضاد الحيوي Doxycycline و الذي أظهر اظهر بنصف قطر

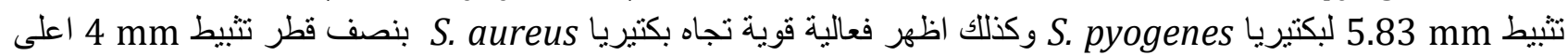

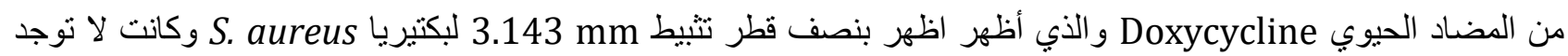

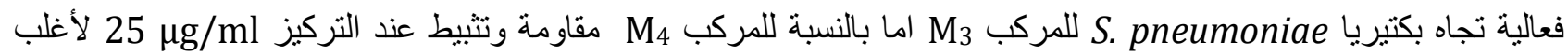

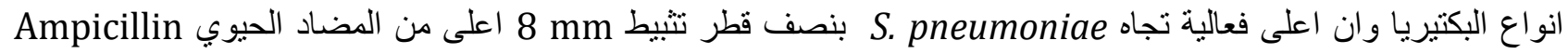

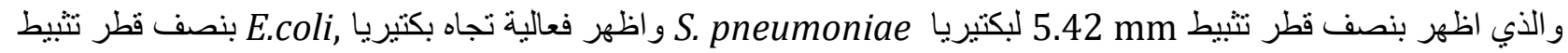

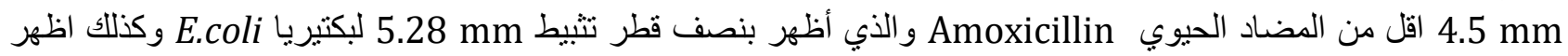

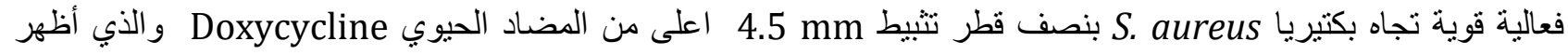

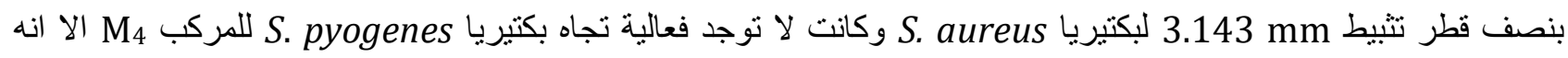

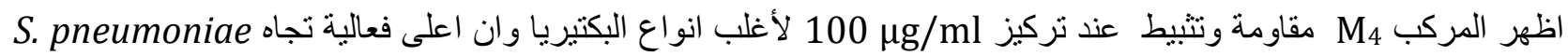

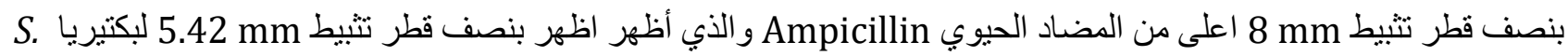

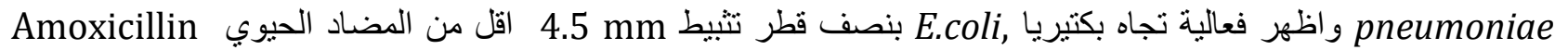

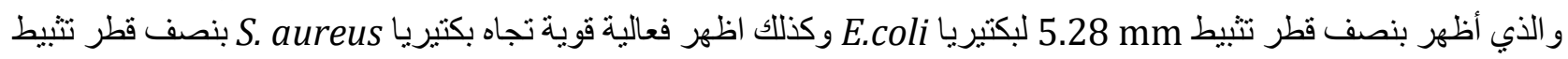

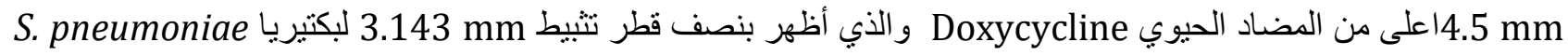

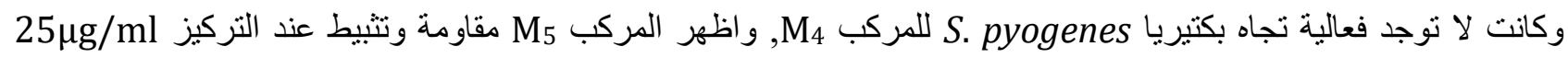

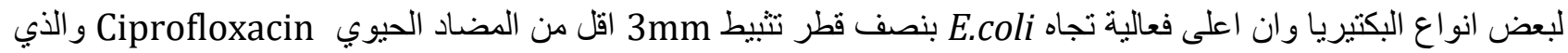

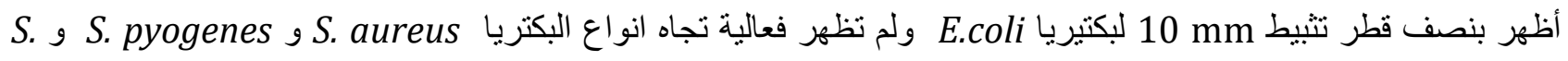

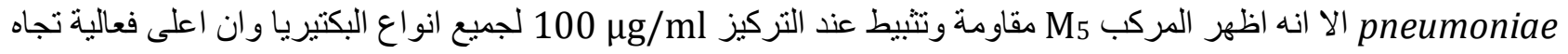
10 mm بن.coli

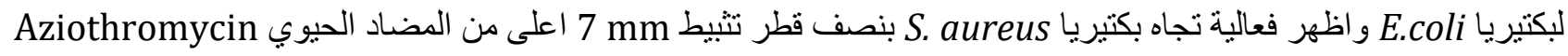

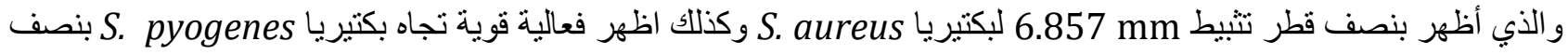

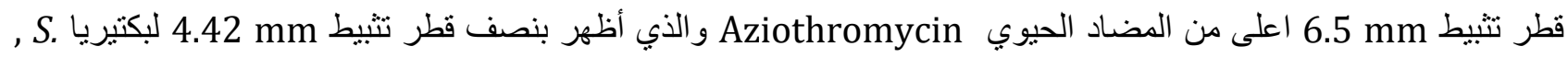
واظهرت فعالية قليلة تجاه بكثيريا Syogenes

و الذي أظهر اظهر بنصف فطر تثبيط 3.714 mm لبكتيريا Soxycycline الجدول 3: الفعالية المضادة للبكتريا للمركبات المحضرة لنمو عدد من الجراثيم السالبة والموجبة (قطر دائرة التثبيط مقاسة بـ

\begin{tabular}{|c|c|c|c|c|c|}
\hline $\begin{array}{c}\text { Comp. } \\
\text { No. }\end{array}$ & $\begin{array}{c}\text { Conc. } \\
\mu \mathrm{g} / \mathrm{ml}\end{array}$ & $\begin{array}{c}E . \\
\text { Coil }\end{array}$ & $\begin{array}{c}\text { S. } \\
\text { Pneumonia }\end{array}$ & $\begin{array}{c}S . \\
\text { Aureus }\end{array}$ & لم). \\
\hline \multirow[t]{6}{*}{ M3 } & 3.125 & 0 & 0 & 0 & 0 \\
\hline & 6.25 & 0 & 0 & 0 & 0 \\
\hline & 12.5 & 0 & 0 & 0 & 0 \\
\hline & 25 & 5.5 & 0 & 0 & 3.5 \\
\hline & 50 & 15.5 & 0 & 3 & 6.75 \\
\hline & 100 & 18 & 0 & 4 & 7.5 \\
\hline \multirow[t]{6}{*}{ M4 } & 3.125 & 0 & 0 & 0 & 0 \\
\hline & 6.25 & 0 & 0 & 0 & 0 \\
\hline & 12.5 & 2.5 & 0 & 0 & 0 \\
\hline & 25 & 3 & 0 & 0 & 0 \\
\hline & 50 & 3.75 & 3.5 & 3 & 0 \\
\hline & 100 & 4.5 & 8 & 4.5 & 0 \\
\hline \multirow[t]{5}{*}{ M5 } & 3.125 & 0 & 0 & 0 & 0 \\
\hline & 6.25 & 0 & 0 & 0 & 0 \\
\hline & 12.5 & 4 & 0 & 0 & 0 \\
\hline & 25 & 4.5 & 2 & 3.4 & 2.5 \\
\hline & 50 & 6 & 3 & 5.5 & 5.5 \\
\hline
\end{tabular}


جدول 4: الفعالية المضادة للبكتريا لمعاملات السبطرة (المضادات الحيوية) في نمو عدد من الجر اثثم السالبة و الموجبة (نصف

\begin{tabular}{cccccc}
$\begin{array}{c}\text { Comp. } \\
\text { No. }\end{array}$ & Name & $\begin{array}{c}\boldsymbol{E} \\
\text { Coil }\end{array}$ & $\begin{array}{c}\boldsymbol{S} \\
\text { pneumonia }\end{array}$ & $\begin{array}{c}\boldsymbol{S} \\
\text { Aureus }\end{array}$ & $\begin{array}{c}\text { S. } \\
\text { pyogenes }\end{array}$ \\
\hline $\mathbf{1}$ & Azithromycin & 5.28 & 8.286 & 6.857 & 8.28 \\
\hline $\mathbf{2}$ & Ciprofloxacin & 10 & 11.86 & 0 & 0 \\
\hline $\mathbf{3}$ & Cephalosporin & 7.75 & 1.429 & 0 & 4 \\
\hline $\mathbf{4}$ & Ampicillin & 9.28 & 1.571 & 1.143 & 4.83 \\
\hline $\mathbf{5}$ & Doxycycline & 9.71 & 3.714 & 3.143 & 5.83 \\
\hline $\mathbf{6}$ & Amoxicillin & 5.57 & 5.42 & 0 & 4.42 \\
\hline
\end{tabular}

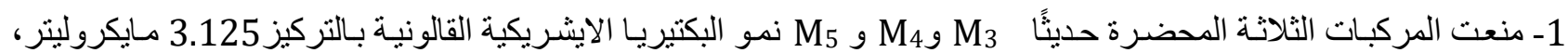

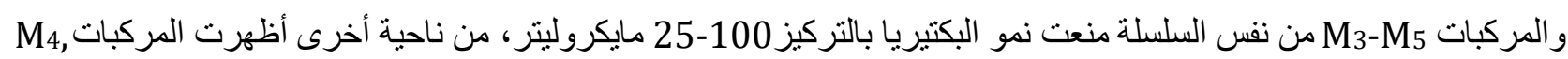

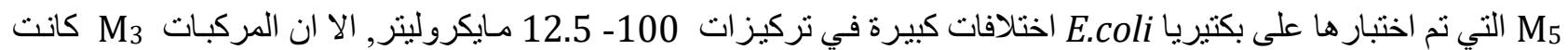

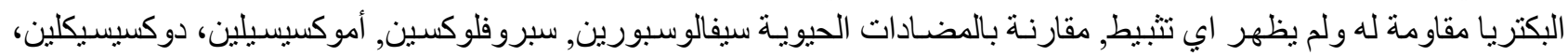

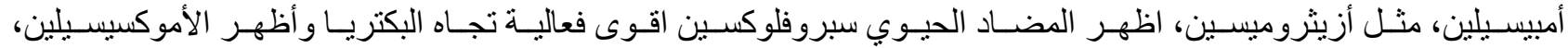

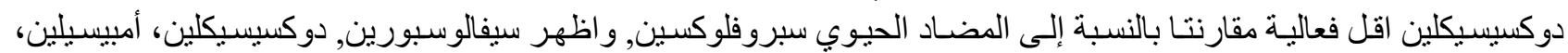
فعالية قليلة جدا للبكتريا لاحظ "الثكل 7 و8" و "الجدول 3 و 4 " قيم الفعالية التثبيطية لبعض المركبات المحضرة و المضسادات

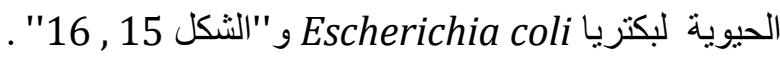

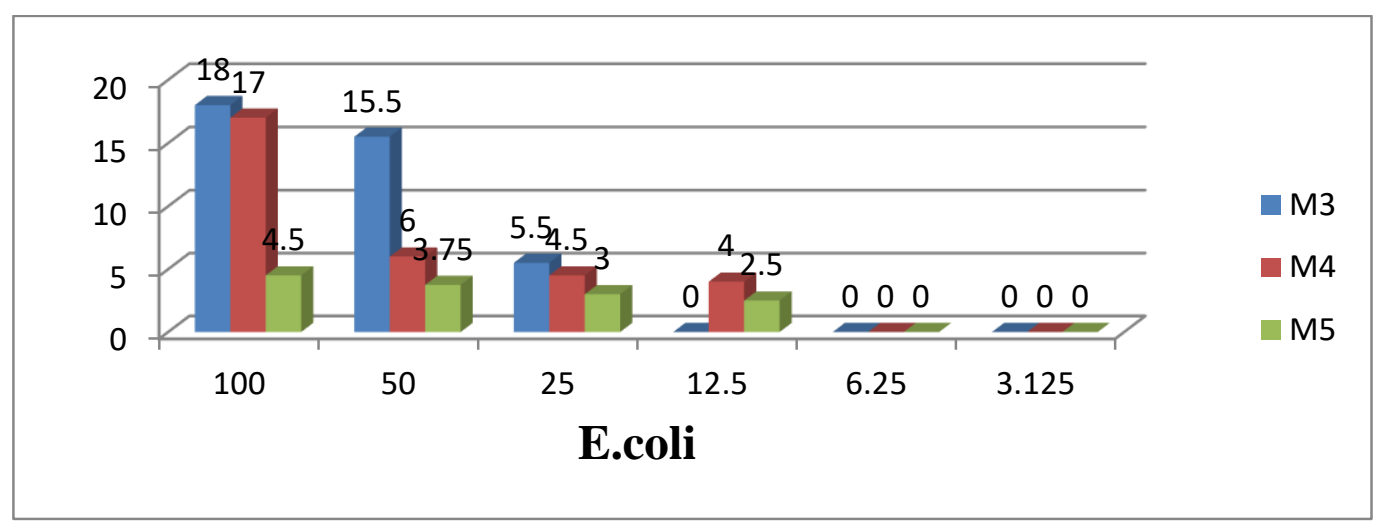

الثكل 7 : قيم الفعالية التثيطية للمركبات تجاه بكتيريا E. coli.

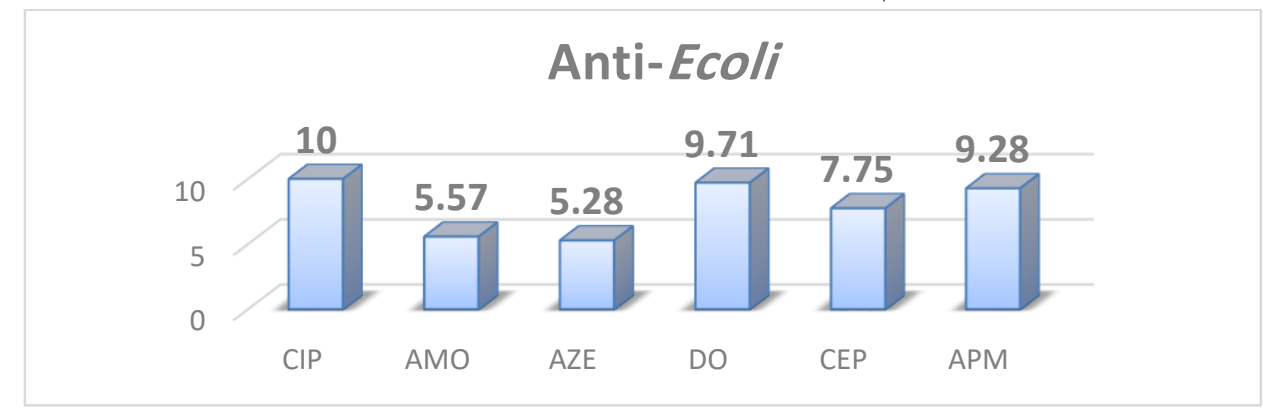

الثكل 8: قيم الفعالية التبيطية لبعض المضادات الحيوية تجاه بكتريا E. coli.

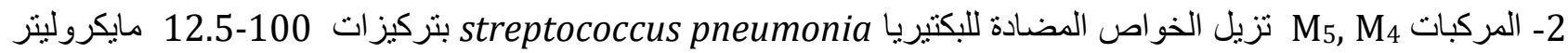

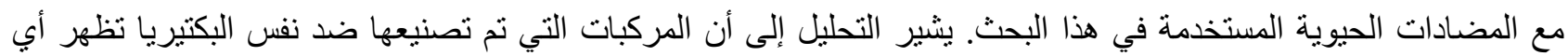
نشاط مضاد للجر اثيم أقل من 100 مايكروليتر. 


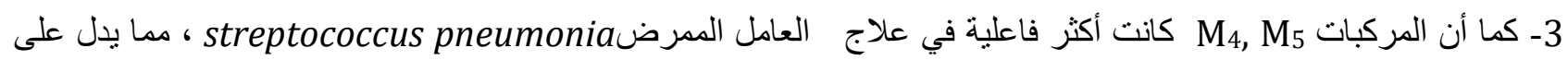

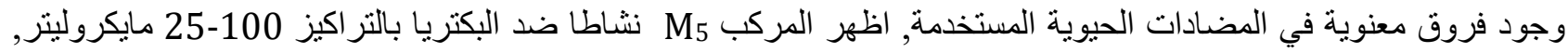

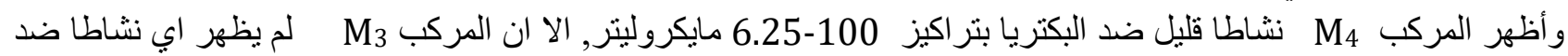

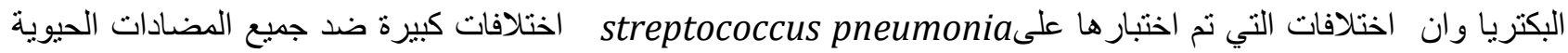

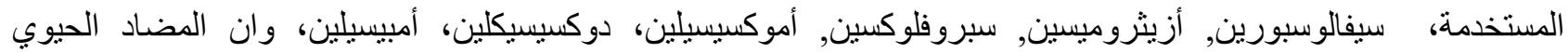
سبروفلوكسين هو اقوى المضادات الحيوية تجاه البكتريا، وان المضاد الحيوي أزيثروميسين هو اقل المضادادين الحينية الحيوية تجاه

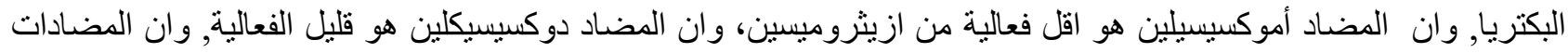

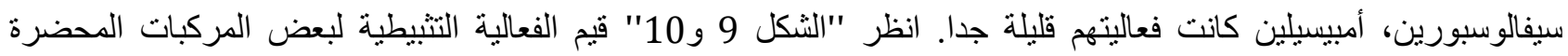
و المضادات الحيوية لبكتريا S. pneumonia و "الجدول 3 و 4 "و "الثكل 15 , 16" :

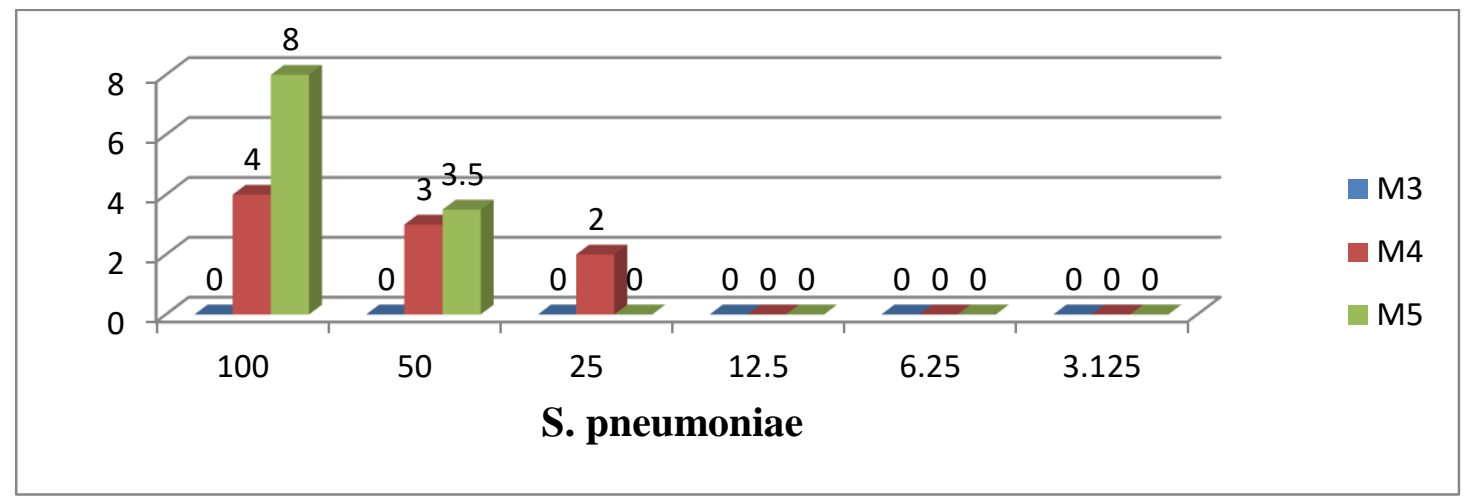

S. pneumonia الثكل 9: قيم الفعالية التثبيةية للمركبات تجاه بكتريا

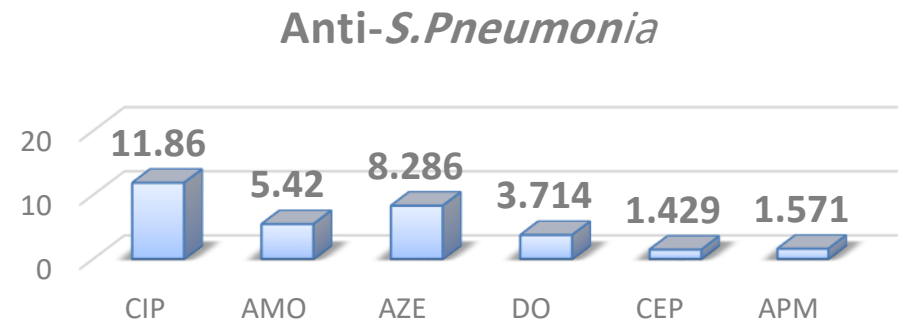

\section{S. pneumonia الثكل 10 : قيم الفعالية التثبيطية للمضادات الحيوية تجاه بكتريا}

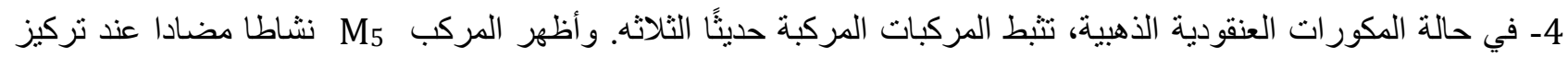
12.5-100مايكروليتر, وان المركبات M3,M4 اظهرت نشاطا ضد البكتيريا عند التر اكيز 100-25 مايكروليتر, في حين ثبطت

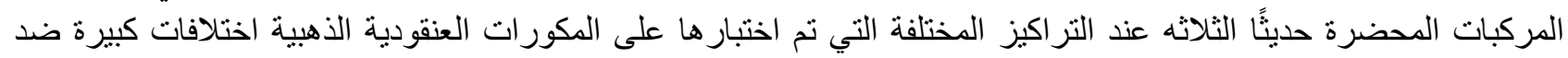

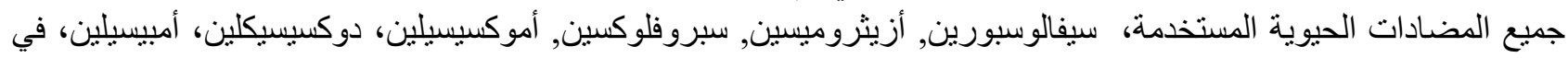

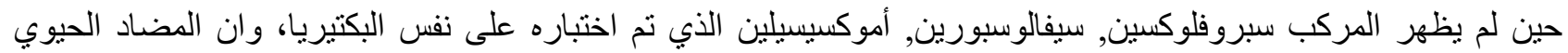

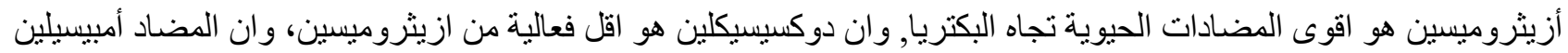

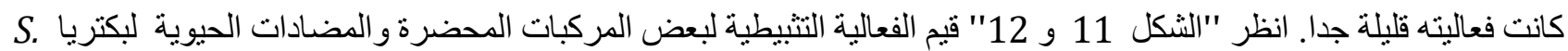

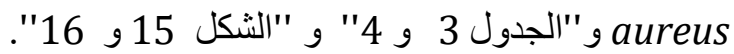

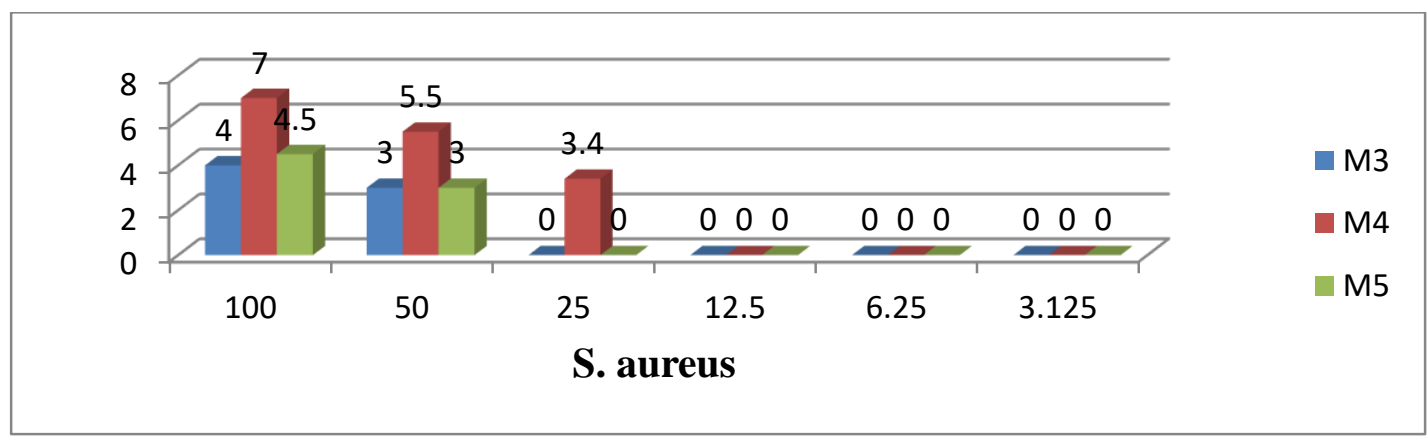

S. aureus الشكل 11 : قيم الفعالية التثبيةية لمركبات تجاه بكتريا 


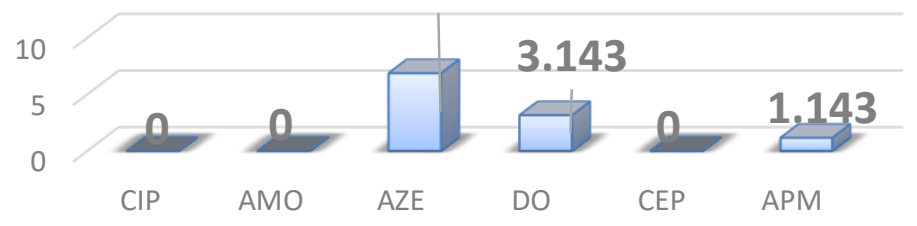

\section{S. aureus الثكل 12 : قيم الفعالية التثبيةية لبعض المضادات الحيوية لبكتريا}

5 - في حالة المكورات العقدية المقيحة، تثبط المركبات المركبة حديثًا الثلاثه تطور هذا العامل الممرض بتركيز يتراوح بين

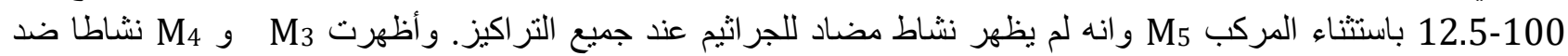

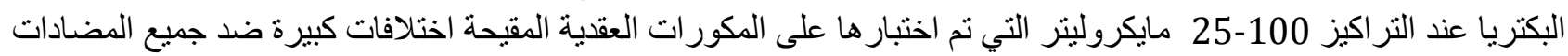

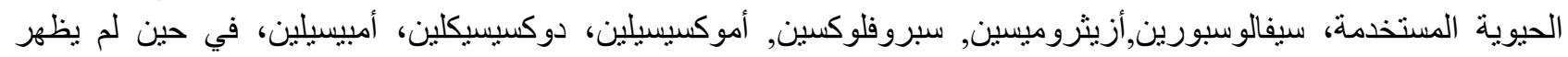

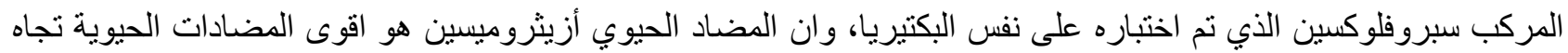

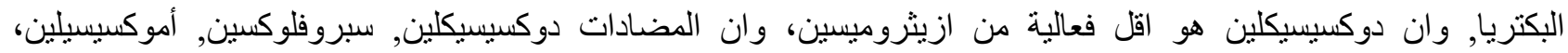

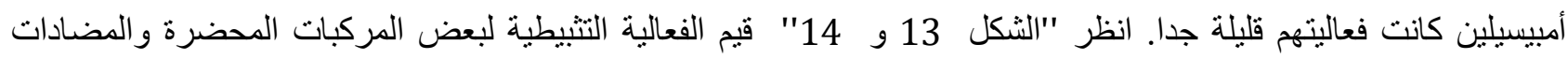

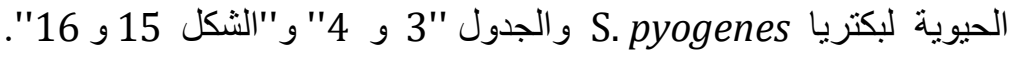

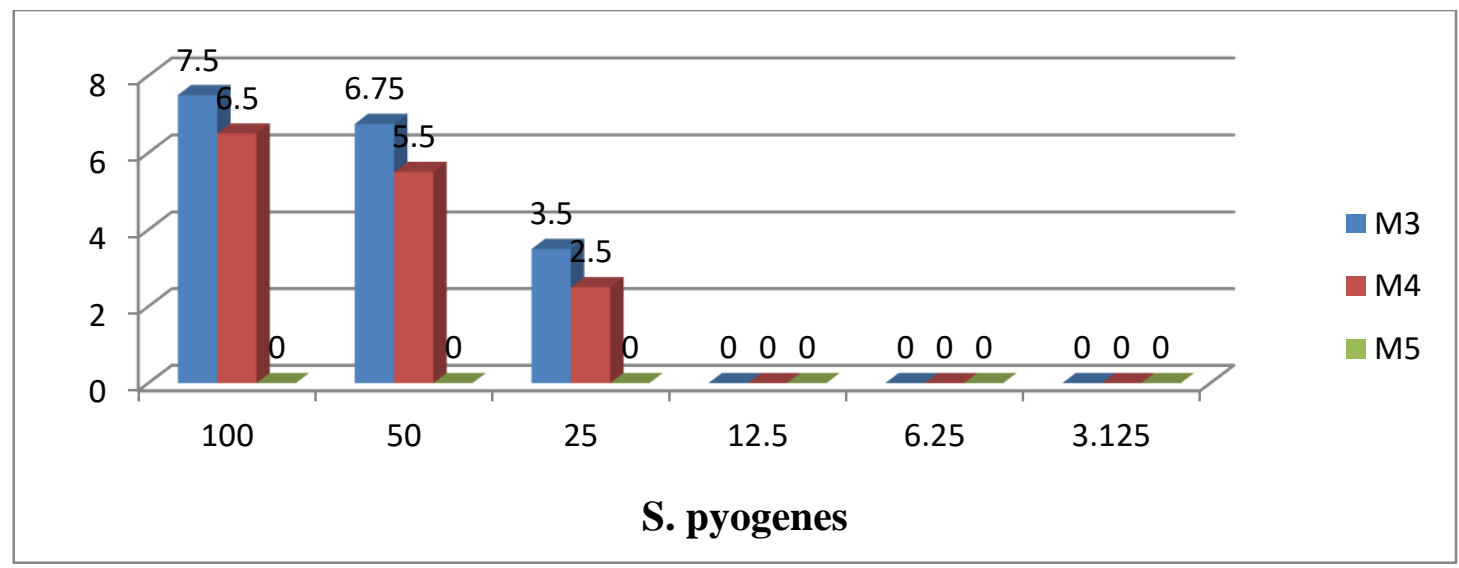

S. pyogenes الثكل 13 : قيم الفعالية التثيطية لمركبات تجاه بكتريا

\section{Anti-Strep.Pyogenes}

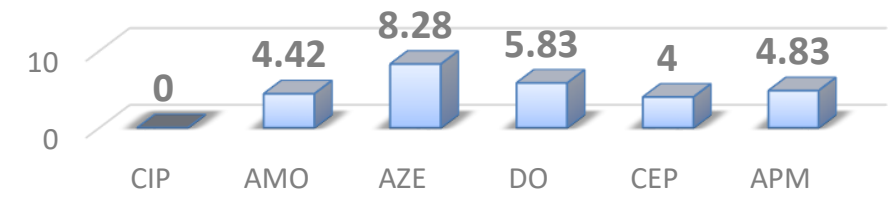

\section{S. pyogenes الثكل 14 : قيم الفعالية التبيطية لبعض المضادات الحيوية لبكتريا}


1- الموجبة لصبغة الكر ام: S. aureus Streptococcus pyogenes E. coli, streptococcus pneumonia: السالبة لصبغة الكر ام 2
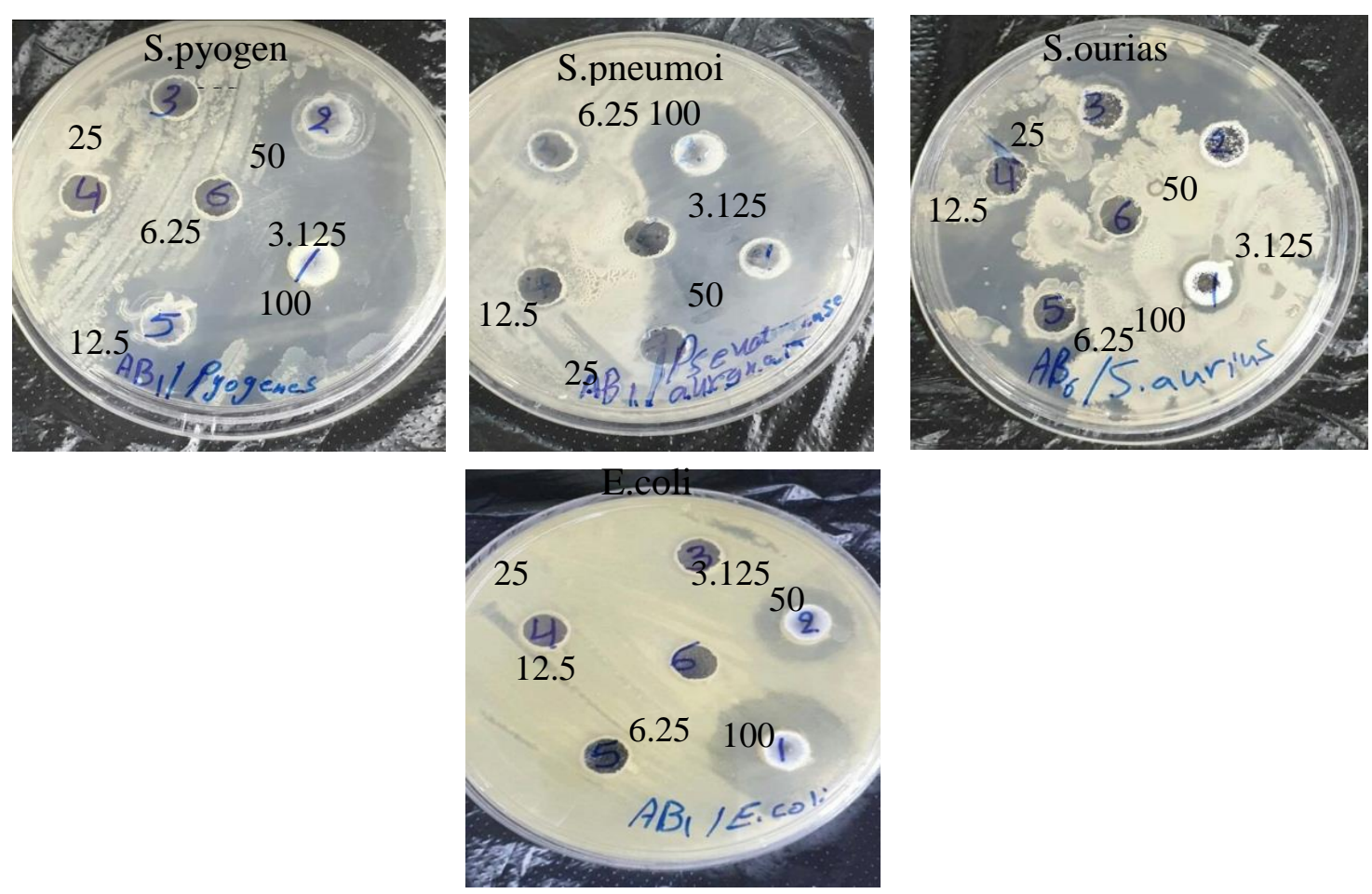

الثكل 15 : منطقة تثبيط المركبات الاصطناعية ضد بكتيريا E.coli و Streptococcus pneumonia و.aureu Streptococcus pyogene
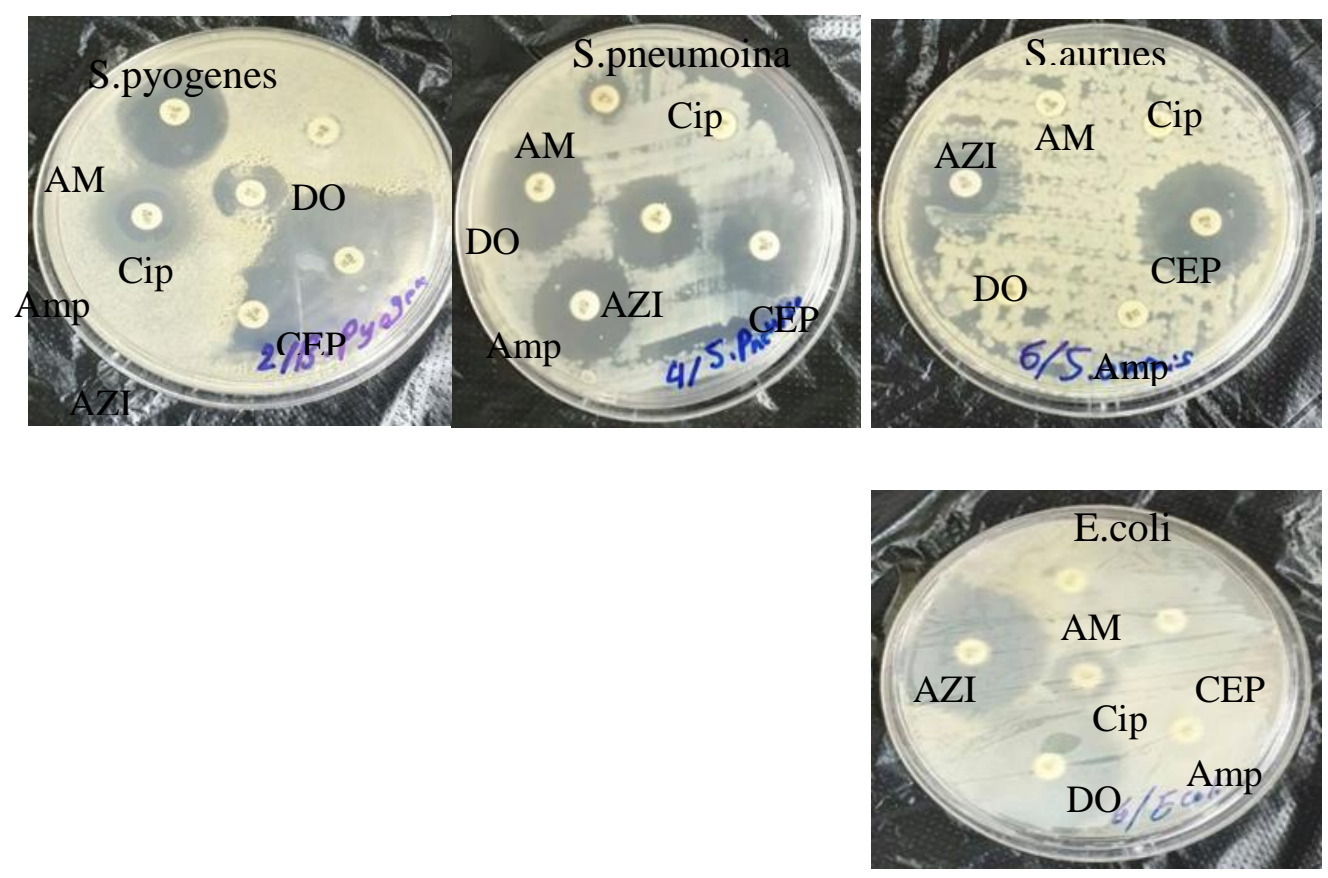

Amoxicillin, Ampicillin, Ciprofloxacin,Cephalosporin, الشكل16 : فعالية تثبيط المضادات الحيوية S. pneumoniae ,E.coli, S. pyogenes,S. aureus ضد بكتريا Azithromycin and Doxycycline 
1. إن الطريقة التقليدية المستخدمة للحصول على المركبات العضوية كانت نتائجها ممتازة، لكونها اقتصادية، إذ أن هذه التقنية

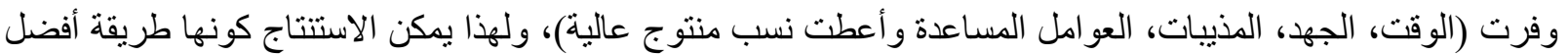

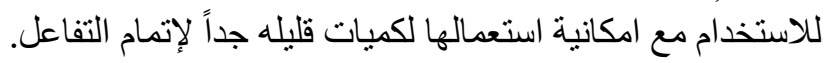

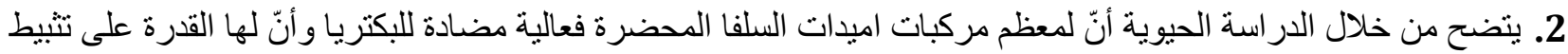
نمو البكتريا، إذ وجد أن لهذه المركبات فعالية بايولوجية عالية، لأن المواد الأولية هي أدوية مستخدمة في الحقول الطبية.

\section{References}

1. Al-Mulla, A. (2017). A review: biological importance of heterocyclic compounds. Der Pharma Chemica, 9(13), 141-147.

2. . Padmavathi, V., Subbaiah, D. R. C. V., Mahesh, K., \& Lakshmi, T. R. (2007). Synthesis and Bioassay of Amino-pyrazolone, Amino-isoxazolone and Amino-pyrimidinone Derivatives. Chemical and Pharmaceutical Bulletin, 55(12), 1704-1709.

3. Giustra, Z. X., Ishibashi, J. S., \& Liu, S. Y. (2016). Homogeneous metal catalysis for conversion between aromatic and saturated compounds. Coordination Chemistry Reviews, 314, 134-181.

4. Mhemeed, A. H. (2019). A review on sulpha drugs (one of the first microbial inhibitors). Int. J. Res. Pharm. Sci.(Madurai, India), 10, 241-244.

5. Maren, T. H. (1976). Relations between structure and biological activity of sulfonamides. Annual Review of Pharmacology and Toxicology, 16(1), 309-327.

6. Boyd, A. E. (1988). Sulfonylurea receptors, ion channels, and fruit flies. Diabetes, 37(7), 847-850.

7. Supuran, C. T. (2017). Carbonic anhydrase inhibition and the management of hypoxic tumors. Metabolites, 7(3), 48.

8. Puccetti, L., Fasolis, G., Vullo, D., Chohan, Z. H., Scozzafava, A., \& Supuran, C. T. (2005). Carbonic anhydrase inhibitors. Inhibition of cytosolic/tumor-associated carbonic anhydrase isozymes I, II, IX, and XII with Schiff's bases incorporating chromone and aromatic sulfonamide moieties, and their zinc complexes. Bioorganic \& Medicinal Chemistry Letters, 15(12), 3096-3101.

9. Supuran, C. T. (2016). How many carbonic anhydrase inhibition mechanisms exist?. Journal of enzyme inhibition and medicinal chemistry, 31(3), 345-360.

10. Carta, F., \& Supuran, C. T. (2013). Diuretics with carbonic anhydrase inhibitory action: a patent and literature review (2005-2013). Expert opinion on therapeutic patents, 23(6), 681-691.

11. Scozzafava, A., Supuran, C. T., \& Carta, F. (2013). Antiobesity carbonic anhydrase inhibitors: a literature and patent review. Expert opinion on therapeutic patents, 23(6), 725-735.

12. Capasso, C., \& Supuran, C. T. (2014). Sulfa and trimethoprim-like drugs-antimetabolites acting as carbonic anhydrase, dihydropteroate synthase and dihydrofolate reductase inhibitors. Journal of enzyme inhibition and medicinal chemistry, 29(3), 379-387.

13. Carta, F., Mannelli, L. D. C., Pinard, M., Ghelardini, C., Scozzafava, A., McKenna, R., \& Supuran, C. T. (2015). A class of sulfonamide carbonic anhydrase inhibitors with neuropathic pain modulating effects. Bioorganic \& medicinal chemistry, 23(8), 1828-1840.

14. Chavan, A. A., \& Pai, N. R. (2007). Sulfonylureas as Dual Acting Agents-Synthesis and Biological Activity. Journal of the Chinese Chemical Society, 54(3), 771-777.

15. Dane, E., Drees, F., Konrad, P., \& Dockner, T. (1962). $\beta$-Dicarbonylverbindungen als Aminoschutzgruppen bei Peptidsynthesen. Angewandte Chemie, 74(21), 873-873. 
16. Supuran, Claudiu. "sulfonamides." Molecules (2017): 22: 1-5.

17. Mok, B. L. (2008). Synthesis of functionalised sulfonamides (Doctoral dissertation, UCL (University College London)).

18. Chan, O. W., Lin, J. J., Hsia, S. H., Lin, C. Y., \& Lin, K. L. (2020). Methylprednisolone pulse therapy as an adjuvant treatment of Streptococcus pneumoniae meningitis complicated by cerebral infarction-a case report and review of the literature. Child's Nervous System, 36(2), 229-233.

19. Griffith, F. (1934). The serological classification of Streptococcus pyogenes. Epidemiology \& Infection, 34(4), 542-584.

20. Apaydın, S., \& Török, M. (2019). Sulfonamide derivatives as multi-target agents for complex diseases. Bioorganic \& medicinal chemistry letters, 29(16), 2042-2050.

21. Shivshankar, S., Chavan, S., \& Vibhute, Y. (2016). Solvent-free synthesis of chalcones and antioxidant activity. Journal of Advanced Chemical Sciences, 373-375.

22. Hoffman, R. V. Structure Determination of Organic Compounds. In Organic Chemistry 332294(Springer,2005). doi:10.1002/047136.ch11.

23. Venkataraman, R. Meera1, Pandiarajan, P.Devi. "Synthesis and biological activity of some novel quinazolinone derivatives." J. Chem. Pharm. Res (2010):2(5):461-474.

24. Donald L. Pavia., Gary M. Lampman a, George S. Kriz and James R. Vyvyan. "Introduction to spectroscopy"(2015):5:225-348.

25. Working, S. \& Tests, S. crossm CLSI Methods Development and Standardization Working Susceptibility Tests. (2018), 56, 1-10 .

26. Genç, Y., Özkanca, R., \& Bekdemir, Y. (2008). Antimicrobial activity of some sulfonamide derivatives on clinical isolates of Staphylococus aureus. Annals of clinical microbiology and antimicrobials, 7(1), 1-6.

27. Özden, S., Atabey, D., Yıldız, S., \& Göker, H. (2005). Synthesis and potent antimicrobial activity of some novel methyl or ethyl 1H-benzimidazole-5-carboxylates derivatives carrying amide or amidine groups. Bioorganic \& medicinal chemistry, 13(5), 1587-1597.

28. Ranjith, P. K., Pakkath, R., Haridas, K. R., \& Kumari, S. N. (2014). Synthesis and characterization of new N-(4-(4-chloro-1H-imidazol-1-yl)-3-methoxyphenyl) amide/sulfonamide derivatives as possible antimicrobial and antitubercular agents. European journal of medicinal chemistry, 71, 354-365..

29. Rehman, H., Qadir, A., Ali, Z., Nazir, S., Zahra, A., \& Shahzady, T. G. (2017). Synthesis and characterization of novel sulfonamides derivatives and their antimicrobial, antioxidant and cytotoxicity evaluation. Bulletin of the Chemical Society of Ethiopia, 31(3), 491-498.

30. Tačić, A., Nikolić, V., Nikolić, L., \& Savić, I. (2017). Antimicrobial sulfonamide drugs. Advanced technologies, 6(1), 58-71. 


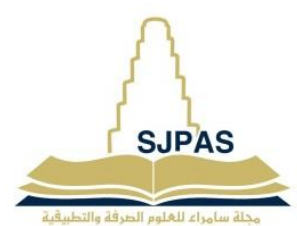

\section{Synthesis, characterization of some sulfonamide derivatives and their anti-bacterial activity}

\section{Maimoonah.H. K. Alesawee ${ }^{1 *}$, Abdulmajeed S. H. Alsamarrai2*and Aseel Mokdad Hatam Abdulwahed $^{3 *}$}

1- Department of Chemistry, College of Education , University of Samarra, Iraq (mymwnthmyd777@gmail.com)

2- Department of Chemistry, College of Applied Sciences, University of Samarra, Iraq

3- Department of Pathology, College of Applied Sciences, University of Samarra , Iraq

\section{Article Information}

Received: $19 / 11 / 2020$

Accepted: 09/01/2021

\section{Keywords:}

Sulphonamide, antibacterial activity, heterocyclic compounds

\begin{abstract}
The research includes preparation of sulfa amide derivatives containing amines and evaluating their biological efficacy. The compounds prepared are sulfa amides. Some of them were diagnosed by means of IR, 1H-NMR NMR-13C techniques, and they assessed the biological effectiveness of the compounds. The measurement was done on the basis of measuring the diameter of inhibition caused by bacterial isolates on the agricultural environment and showed high inhibition of some compounds, some of which were few against bacteria. One of the compounds showed great activity against E.coli with an inhibition radius of $18 \mathrm{~mm}$ while the other compound showed efficacy against E.coli and S. aureues. Inhibition radii of 17 and $7 \mathrm{~mm}$, respectively. Another compound showed activity against S. pneumonia with an inhibition radius of $8 \mathrm{~mm}$. Others, however, did not show efficacy against $S$. pneumonia and S. pyogenes.
\end{abstract}

